\title{
Direct numerical simulations of turbulent flow over various riblet shapes in minimal-span channels
}

\author{
S. Endrikat · D. Modesti · M. MacDonald · \\ R. García-Mayoral · N. Hutchins · D. Chung
}

Received: date / Accepted: date

\begin{abstract}
Riblets reduce skin-friction drag until their viscous-scaled size becomes large enough for turbulence to approach the wall, leading to the breakdown of drag-reduction. In order to investigate inertial-flow mechanisms that are responsible for the breakdown, we employ the minimal-span channel concept for cost-efficient direct numerical simulation (DNS) of rough-wall flows (MacDonald et al., J. Fluid Mech., vol. 816, 2017, pp. 5-42). This allows us to investigate six different riblet shapes and various viscous-scaled sizes for a total of 21 configurations. We verify that the small numerical domains capture all relevant physics by varying the box size and by comparing to reference data from full-span channel flow. Specifically, we find that, close to the wall in the spectral region occupied by dragincreasing Kelvin-Helmholtz rollers (García-Mayoral \& Jiménez, J. Fluid Mech., vol. 678, 2011, pp. 317-347), the energy-difference relative to smooth-wall flow is not affected by the narrow domain, even though these structures have large spanwise extents. This allows us to evaluate the influence of the Kelvin-Helmholtz instability by comparing fluctuations of wall-normal and streamwise velocity, pressure and a passive scalar over riblets of different shapes and viscous-scaled sizes to those over a smooth wall. We observe that triangular riblets with a tip angle $\alpha=30^{\circ}$ and blades appear to support the instability, whereas triangular riblets with $\alpha=60^{\circ}-90^{\circ}$ and trapezoidal riblets with $\alpha=30^{\circ}$ show little to no evidence of Kelvin-Helmholtz rollers.
\end{abstract}

Keywords Riblets $\cdot$ minimal-span channel $\cdot$ DNS $\cdot$ Kelvin-Helmholtz

\section{Drag-reduction performance of riblets}

Riblets are small streamwise-aligned grooves on a surface that have been shown to reduce skin-friction drag compared to a smooth wall [41,40,28,3]. The skin-friction coefficient

S. Endrikat $\cdot$ D. Modesti $\cdot$ N. Hutchins $\cdot$ D. Chung

Department of Mechanical Engineering, University of Melbourne, Victoria 3010, Australia

E-mail: sendrikat@student.unimelb.edu.au

M. MacDonald

Department of Mechanical Engineering, University of Auckland, Auckland 1010, New Zealand

R. García-Mayoral

Department of Engineering, University of Cambridge, Cambridge CB2 1PZ, United Kingdom 
$C_{f}=2 / U_{\delta}^{+2}$ is defined by the friction-scaled mean streamwise velocity $U^{+} \equiv U / u_{\tau}$ at the half-channel or boundary-layer height $\delta$. The superscript + denotes viscous scaling with the kinematic fluid viscosity $v$ and friction velocity $u_{\tau} \equiv \sqrt{\tau_{w} / \rho}$, where $\rho$ is the fluid density and $\tau_{w}$ the wall shear stress (drag per unit plan area), such that $C_{f}=2 \tau_{w} /\left(\rho U_{\delta}^{2}\right)$. Drag reduction of a riblet surface compared to a smooth wall is commonly given by the relative change of the skin-friction coefficients DR $\equiv 1-C_{f} / C_{f \text {,smooth }}$. However, $C_{f \text {,smooth }}$, and therefore DR, degrades with increasing Reynolds number [37]. An alternative, Reynolds number independent measure of the drag-change is given by the decrement of the viscous-scaled mean streamwise velocity, $\Delta U^{+} \equiv U_{\text {smooth }}^{+}-U^{+}$, at matched heights in the outer layer of two flows with the same friction Reynolds number $R e_{\tau} \equiv \delta^{+}=\delta u_{\tau} / v$. For example, DR $\approx-0.1 \Delta U^{+}$at $R e_{\tau}=395$, which is accessible through DNS, but DR $\approx-0.06 \Delta U^{+}$at flight conditions with $R e_{\tau}=50000$, based on the conversion derived by Spalart \& McLean [37].

The flow mechanism responsible for drag-reduction by riblets is well understood and can be explained by the concept of protrusion heights in the limit of vanishingly small riblets. The streamwise mean-flow reaches an average depth below the riblet crest given by the longitudinal protrusion height $\ell_{U}^{+}$, that depends on the groove shape and size [2]. Turbulent lateral flow is obstructed by the riblets and therefore only penetrates the groove to a depth given by $\ell_{T}^{+}<\ell_{U}^{+}$[28]. Luchini [27] points out that a reference smooth wall should be considered at the height given by $\ell_{T}^{+}$for the two flows to be similar, i.e. that their total stresses match at every height. This placement of the reference wall therefore provides the correct measure of the drag-change $\Delta U^{+}$. At the height of the reference smooth-wall, the riblet flow has the velocity $U^{+}=\ell_{U}^{+}-\ell_{T}^{+}$[27], because the slope $\mathrm{d} U^{+} / \mathrm{d} z^{+} \approx 1$ in the viscous sublayer. The velocity difference compared to the smooth wall flow extends into the logarithmic layer, where it describes the drag-change $\Delta U^{+}=\ell_{U}^{+}-\ell_{T}^{+}$[27]. However, this relation between the protrusion heights and $\Delta U^{+}$is only valid for vanishingly small riblets and as their viscousscaled size increases, drag characteristics change significantly. The drag-change optimum of traditional riblet shapes was empirically shown to scale with the viscous-scaled groove cross-section $A_{g}^{+}$and is obtained for riblet sizes $\ell_{g}^{+} \equiv \sqrt{A_{g}^{+}} \approx 11$ [14].

For larger riblets, drag increases and ultimately surpasses smooth-wall drag, because the flow in and around the grooves becomes less dominated by viscosity as different inertial flow effects contribute to drag. The spanwise component of streamwise vortices is deflected downward by the riblet tips, creating secondary flows that transport momentum towards the wall [17]. Particularly for widely spaced riblets, the streamwise vortices and their secondary flows sweep streamwise momentum towards the large wetted surface area [7]. Furthermore, Kelvin-Helmholtz rollers have been shown to augment wall-normal momentum transport towards the wall above blade riblets, leading to increased drag [14]. These rollers evolve around an inflection point in the profile of mean streamwise velocity that is created between the slower flow in the groove and the faster bulk flow above. Kelvin-Helmholtz rollers are well known in free shear flows [9], but have also been observed in wall-bounded flows over porous surfaces $[25,4,18]$ and vegetation canopies $[35,34,33,36]$ that allow wall-normal velocity fluctuations. For riblets, fewer data are available, but García-Mayoral \& Jiménez $[14,15]$ note that the Kelvin-Helmholtz instability affects a distinct spectral region in the flow over blade riblets. By comparing 2D spectra in DNS at heights of less than 30 viscous units above the riblet crest to smooth-wall flow, they find that Kelvin-Helmholtz rollers have an average streamwise periodicity in the range $65 \lesssim \lambda_{x}^{+} \lesssim 290$ and that they are spanwise coherent with wavelengths $\lambda_{y}^{+} \gtrsim 130$. In this study we will more conservatively only consider structures with $\lambda_{y}^{+} \gtrsim 250$ to separate them more clearly from other near-wall turbulence (details in $\S 4.3$ ). 
García-Mayoral \& Jiménez [14] evaluate the momentum transport by Reynolds stresses due to Kelvin-Helmholtz rollers to quantify the drag penalty associated with the instability. They demonstrate that drag from Kelvin-Helmholtz rollers becomes more significant with increasing size of blade riblets, which coincides with the breakdown of drag reduction for $\ell_{g}^{+} \gtrsim 11$. Therefore, Kelvin-Helmholtz rollers contribute to the drag increase of large blade riblets [14,15], as also observed for vegetation canopies [34,36] and porous surfaces [18]. However, it remains to be seen if Kelvin-Helmholtz rollers develop above all riblets, because studies of plant canopies and porous substrates also show that the Kelvin-Helmholtz instability only develops if the surface creates the necessary drag and inflectional velocity profile $[35,34,33]$. In fact, in the present study, we show that the appearance of Kelvin-Helmholtz rollers over riblets is likewise not universal, but instead dependent on the riblet geometry. We visualise a dependence of Kelvin-Helmholtz rollers on the groove shape (§ 4.2) and quantify the contribution of Kelvin-Helmholtz rollers in spectral space across distances from the wall for six different riblet geometries (§ 4.4).

In order to study the drag characteristics of a broad range of riblet cross sections and sizes, we conduct DNS in minimal-span channels, which reduce the computational cost in exchange for unphysical results towards the top of the domain. Minimal-span channels have previously been used to investigate the flow over generic roughness [30], but KelvinHelmholtz rollers, having a large spanwise extent [15], may pose a unique challenge for spanwise narrow domains. In $\S 3$, we therefore rigorously investigate the velocity spectrum at different scales, wall-normal distances and for varying riblet geometries, to determine the limitations of minimal-span channels for riblet flows.

\section{Numerical setup of minimal-span channel flow}

We investigate the turbulent flow over six different riblet shapes at various viscous-scaled sizes (table 2) using DNS in minimal-span channels. We consider triangular riblets with varying tip angle $\alpha=30^{\circ}, 60^{\circ}, 90^{\circ}$ (case names $\mathrm{T} 3 s^{+}, \mathrm{T} 6 s^{+}, \mathrm{T} 9 s^{+}$), asymmetric triangular riblets with $\alpha=63.4^{\circ}\left(\mathrm{AT}^{+}\right.$, sketched in table 2), trapezoidal riblets with $\alpha=30^{\circ}\left(\mathrm{TA}^{+}\right)$, and blade riblets with a spacing-to-thickness ratio $s / t=5\left(\mathrm{BLs}^{+}\right)$. The simulations for two large triangular riblets (T321 and T950) are repeated in channels of varying width to study minimal-span channel effects on the flow. The case T950 is further repeated with different mesh resolutions to verify results. Domain and simulation parameters for all corresponding smooth-wall flows are given in table 2 .

We employ the incompressible second-order accurate finite volume DNS solver Cliff by Cascade Technologies Inc. $[19,20]$ to solve the Navier-Stokes equations and the transport equation of a passive scalar $\theta$ that can be interpreted as temperature fluctuation

$$
\begin{aligned}
\frac{\partial \mathbf{u}}{\partial t}+\nabla \cdot(\mathbf{u u}) & =-\frac{1}{\rho} \nabla p+v \nabla^{2} \mathbf{u}-\frac{1}{\rho} \frac{\mathrm{d} P}{\mathrm{~d} x} \mathbf{e}_{\mathbf{x}}, \\
\nabla \cdot \mathbf{u} & =0, \\
\frac{\partial \theta}{\partial t}+\nabla \cdot(\mathbf{u} \theta) & =v_{\theta} \nabla^{2} \theta-u \frac{\mathrm{d} \Theta}{\mathrm{d} x} .
\end{aligned}
$$

The velocity $\mathbf{u}$ has components $u, v$ and $w$ in the streamwise $(x)$, spanwise $(y)$ and wallnormal $(z)$ directions respectively and $t$ denotes time. We solve for the $x$-y-periodic component of pressure $p$, while the constant mean $\mathrm{d} P / \mathrm{d} x$ drives the flow in the streamwise direction along the unit vector $\mathbf{e}_{\mathbf{x}}$. The spanwise-averaged channel-depth is $\delta$ and therefore, the spatially and temporally averaged wall-shear stress per unit plan area integrates to 


\begin{tabular}{|c|c|c|c|c|c|c|c|c|c|c|c|c|c|c|c|c|c|}
\hline \multirow{5}{*}{$y \longleftarrow \uparrow_{x}^{z}$} & Case & $s^{+}$ & $k^{+}$ & $\ell_{g}^{+}$ & $\alpha$ & $\Delta x^{+}$ & $\Delta y^{+}$ & $n_{s}$ & $\Delta z^{+}$ & $\left(\begin{array}{l}\Delta t^{+} \\
\left(\times 10^{3}\right)\end{array}\right.$ & $L_{x}^{+}$ & $L_{y}^{+}$ & $\delta^{+}$ & $\delta^{\prime+}$ & $L_{t} u_{\tau} / \delta$ & $\Delta U^{+} \pm \zeta^{+}$ & $\begin{array}{c}\varepsilon^{f^{+}} \\
\left(\times 10^{3}\right)\end{array}$ \\
\hline & T310 & 10.1 & 18.8 & 9.75 & $30.0^{\circ}$ & 6.0 & $0.057-1.5$ & 29 & $0.033-7.0$ & 15.8 & 1027 & 252 & 395.0 & 386.3 & 69.3 & $-0.77 \pm 0.12$ & 6.1 \\
\hline & $\mathrm{T} 321$ & 21.1 & 39.4 & 20.4 & $30.0^{\circ}$ & 6.0 & $0.12-3.2$ & 29 & $0.023-6.9$ & 15.8 & 1027 & 253 & 395.0 & 376.9 & 65.0 & $+0.83 \pm 0.12$ & 5.4 \\
\hline & $\mathrm{T} 321 \mathrm{~W}$ & 21.1 & 39.4 & 20.4 & $30.0^{\circ}$ & 6.0 & $0.12-3.2$ & 29 & $0.023-6.9$ & 15.8 & 1027 & 443 & 395.0 & 376.9 & 25.8 & $+1.05 \pm 0.19$ & 4.6 \\
\hline & T333 & 33.3 & 62.2 & 32.2 & $30.0^{\circ}$ & 6.0 & $0.83-3.4$ & 41 & $0.20-8.5$ & 25.0 & 2000 & 600 & 1000.0 & 971.4 & 19.1 & $+2.75 \pm 0.10$ & 8.4 \\
\hline Symmetric triangular & T635 & 35.0 & 30.3 & 23.0 & $60.0^{\circ}$ & 6.0 & $0.16-4.9$ & 33 & $0.014-4.7$ & 23.7 & 1027 & 245 & 395.0 & 383.1 & 93.5 & $+0.64 \pm 0.10$ & 4.1 \\
\hline \multirow{6}{*}{$k \sqrt{2} y^{\alpha}$} & T919 & 19.2 & 9.6 & 9.60 & $90.0^{\circ}$ & 6.0 & $0.11-2.9$ & 29 & $0.047-7.1$ & 47.4 & 1027 & 250 & 395.0 & 392.2 & 127 & $-0.61 \pm 0.08$ & 4.7 \\
\hline & T950W & 50.0 & 25.0 & 25.0 & $90.0^{\circ}$ & 6.0 & $0.30-7.1$ & 33 & $0.029-7.0$ & 47.4 & 1027 & 450 & 395.0 & 387.8 & 93.7 & $+0.79 \pm 0.10$ & 2.5 \\
\hline & T950 & 50.0 & 25.0 & 25.0 & $90.0^{\circ}$ & 6.0 & $0.30-7.1$ & 33 & $0.029-7.0$ & 47.4 & 1027 & 250 & 395.0 & 387.8 & 95.0 & $+0.78 \pm 0.10$ & 7.8 \\
\hline & T950N & 50.0 & 25.0 & 25.0 & $90.0^{\circ}$ & 6.0 & $0.30-7.1$ & 33 & $0.029-7.0$ & 47.4 & 1027 & 150 & 395.0 & 387.8 & 177 & $+0.56 \pm 0.09$ & 5.4 \\
\hline & T950NC & 50.0 & 25.0 & 25.0 & $90.0^{\circ}$ & 8.0 & $0.46-8.1$ & 25 & $0.045-9.3$ & 98.8 & 1027 & 150 & 395.0 & 387.8 & 345 & $+0.54 \pm 0.07$ & 5.2 \\
\hline & T950NVC & 50.0 & 25.0 & 25.0 & $90.0^{\circ}$ & 11.9 & $0.65-8.9$ & 21 & $0.083-14$ & 158 & 1027 & 150 & 395.0 & 387.8 & 523 & $+0.56 \pm 0.05$ & 5.9 \\
\hline \multirow{3}{*}{ Asymmetric triangular } & AT15 & 14.7 & 7.4 & 7.36 & $63.4^{\circ}$ & 6.5 & $0.23-2.0$ & 26 & $0.40-5.8$ & 47.4 & 1027 & 250 & 395.0 & 392.6 & 45.4 & $-0.49 \pm 0.14$ & 3.1 \\
\hline & AT19 & 19.2 & 9.6 & 9.62 & $63.4^{\circ}$ & 6.5 & $0.37-1.9$ & 28 & $0.40-5.8$ & 47.4 & 1027 & 250 & 395.0 & 391.9 & 121 & $-0.50 \pm 0.09$ & 3.4 \\
\hline & AT31 & 31.3 & 15.6 & 15.6 & $63.4^{\circ}$ & 6.5 & $0.15-4.3$ & 55 & $0.40-5.7$ & 47.4 & 1027 & 250 & 395.0 & 390.0 & 47.5 & $-0.32 \pm 0.14$ & 7.2 \\
\hline & AT42 & 41.7 & 20.8 & 20.8 & $63.4^{\circ}$ & 6.5 & $0.45-2.7$ & 46 & $0.40-4.9$ & 47.4 & 1027 & 250 & 395.0 & 388.3 & 117 & $+0.22 \pm 0.09$ & 2.4 \\
\hline & AT50 & 50.0 & 25.0 & 25.0 & $63.4^{\circ}$ & 6.5 & $0.32-1.7$ & 63 & $0.40-4.4$ & 47.4 & 1027 & 250 & 395.0 & 387.0 & 60.3 & $+0.49 \pm 0.12$ & 1.6 \\
\hline \multirow{3}{*}{ Trapezoidal ${ }_{S}$} & TA18 & 17.9 & 8.9 & 11.8 & $30.0^{\circ}$ & 6.0 & $0.27-3.0$ & 27 & $0.31-7.0$ & 47.4 & 2054 & 250 & 395.0 & 389.6 & 50.1 & $-1.06 \pm 0.10$ & 4.2 \\
\hline & TA50 & 50.0 & 25.0 & 32.9 & $30.0^{\circ}$ & 6.0 & $0.76-3.9$ & 27 & $0.31-7.1$ & 47.4 & 2054 & 250 & 395.0 & 379.8 & 45.7 & $+1.76 \pm 0.10$ & 5.9 \\
\hline & TA63 & 62.5 & 31.3 & 41.1 & $30.0^{\circ}$ & 6.0 & $0.95-4.9$ & 27 & $0.31-7.1$ & 47.4 & 2054 & 250 & 395.0 & 376.0 & 46.7 & $+2.47 \pm 0.10$ & 7.3 \\
\hline \multirow[t]{2}{*}{ Blade } & & & & & $s / t$ & & & & & & & & & & & & \\
\hline & BL20 & 20.3 & 10.1 & 12.8 & 5.0 & 6.0 & $0.51-2.1$ & 41 & $0.27-6.1$ & 47.4 & 1027 & 264 & 395.0 & 388.5 & 94.6 & $-0.60 \pm 0.10$ & 3.0 \\
\hline & BL33 & 33.3 & 16.7 & 21.1 & 5.0 & 6.0 & $0.83-3.4$ & 41 & $0.28-6.3$ & 47.4 & 1027 & 266 & 395.0 & 384.4 & 94.6 & $+0.58 \pm 0.10$ & 3.0 \\
\hline & BL39 & 39.0 & 19.5 & 24.7 & 5.0 & 6.0 & $0.97-3.9$ & 41 & $0.29-6.5$ & 47.4 & 1027 & 273 & 395.0 & 382.5 & 93.3 & $+1.19 \pm 0.10$ & 3.1 \\
\hline & BL49 & 49.0 & 24.5 & 31.0 & 5.0 & 6.0 & $1.2-4.9$ & 41 & $0.30-6.7$ & 47.4 & 1027 & 294 & 395.0 & 379.3 & 118 & $+1.84 \pm 0.09$ & 2.9 \\
\hline
\end{tabular}

Table 1 Surface parameters, spacings of the numerical mesh $\Delta^{+}$and domain sizes $L^{+}$. Close to the wall, spanwise meshes have $n_{s}$ nodes per riblet period. We measure the half-channel height $\delta^{+}$from the mean riblet height and $\delta^{\prime+}$ from the virtual origin. Statistics are averaged in the time interval $L_{t}$ to obtain an uncertainty in $\Delta U^{+}$given by $\zeta^{+}$ and the convergence error $\varepsilon^{\prime+}$ given by equation (9). W indicates a wide, and $\mathrm{N}$ a narrow channel. $\mathrm{F}$ stands for a fine mesh and (V)C for (very) coarse. 


$\begin{array}{lccccccccc}\text { Case } & \Delta x^{+} & \Delta y^{+} & \Delta z^{+} & \begin{array}{c}\Delta t^{+} \\ \left(\times 10^{3}\right)\end{array} & L_{x}^{+} & L_{y}^{+} & \delta^{+} & L_{t} u_{\tau} / \delta & \begin{array}{c}\varepsilon^{+} \\ \left(\times 10^{3}\right)\end{array} \\ \text { SFull } & 6.5 & 3.2 & 0.27-4.4 & 83.0 & 2482 & 1241 & 395.0 & 34.9 & 2.3 \\ \text { SW } & 6.0 & 3.0 & 0.21-4.7 & 79.0 & 1027 & 450 & 395.0 & 95.2 & 3.7 \\ \text { S } & 6.0 & 3.0 & 0.21-4.7 & 79.0 & 1027 & 250 & 395.0 & 173 & 3.6 \\ \text { SNF } & 4.0 & 2.3 & 0.21-4.7 & 86.9 & 1027 & 150 & 395.0 & 152 & 4.1 \\ \text { SN } & 6.0 & 3.0 & 0.31-7.1 & 98.8 & 1027 & 150 & 395.0 & 533 & 4.5 \\ \text { SNC } & 8.0 & 3.9 & 0.42-9.5 & 158 & 1027 & 150 & 395.0 & 350 & 5.9 \\ \text { SNVC } & 11.9 & 4.7 & 0.64-14 & 296 & 1027 & 150 & 395.0 & 402 & 11 \\ \text { SH } & 6.0 & 3.0 & 0.32-8.8 & 80.0 & 2000 & 600 & 1000.0 & 58.5 & 3.3\end{array}$

Table 2 Smooth wall simulation parameters: spacings of the numerical mesh $\Delta^{+}$, domain sizes $L^{+}$and halfchannel height $\delta^{+}$. Statistics are averaged in the time interval $L_{t}$ to obtain the convergence error $\varepsilon^{\prime+}$ given by (9). W indicates a wide, and $\mathrm{N}$ a narrow channel. F stands for a fine mesh and (V)C for (very) coarse.

$\tau_{w} / \rho=-(\delta / \rho) \mathrm{d} P / \mathrm{d} x$ for smooth-wall and riblet cases alike. Analogously, the temperature field has an $x$-y-periodic component $\theta$ and mean $\Theta$. The spatially and temporally averaged heat flux into the wall is thus given by $q_{w} /\left(\rho c_{p}\right)=-\delta U_{\text {bulk }} \mathrm{d} \Theta / \mathrm{d} x$, where $U_{\text {bulk }}$ is the volume-averaged streamwise velocity and $c_{p}$ is the specific heat at constant pressure. In our setup, $\mathrm{d} \Theta / \mathrm{d} x<0$ such that the wall serves as a heat sink in analogy to momentum.

Computational domains (figure $1 b$ ) are rectangular open channels with a symmetry boundary condition at the top such that the domain height equals the half-channel height $\delta$. The no-slip bottom wall is either smooth for reference or it has streamwise aligned riblets. For the passive scalar, we set the isothermal boundary condition on the fluctuation $\theta=0$ at the wall. Periodicity is applied in both horizontal directions $x$ and $y$. All quantities are nondimensionalised using the friction velocity $u_{\tau}$, half-channel height $\delta$, kinematic viscosity $v$ and friction temperature $\theta_{\tau}=q_{w} /\left(\rho c_{p} u_{\tau}\right)$. The friction Reynolds number is fixed at $R e_{\tau}=395$ and for one case $R e_{\tau}=1000$. The Prandtl number $\operatorname{Pr}=0.7$ sets the thermal conductivity (scalar diffusivity) $v_{\theta}=v / P r$.

\subsection{Definition of turbulent fluctuations}

We define turbulent fluctuations of any given scalar field $\phi \in\{u, v, w, p, \theta\}$ as $\phi^{\prime}(x, y, z, t)=$ $\phi(x, y, z, t)-\phi^{x r t}(y, z)$, i.e. as deviations from the streamwise $(x)$, riblet-period $(r)$ and temporal $(t)$ average $\phi^{x r t}$. We use the overbar to denote plane averages across $x, y$ and $t$ at a given height above the riblet tips $z \gtrsim z_{t}$ and apply it to products of two fluctuating quantities $\overline{\phi^{\prime} \psi^{\prime}}$ (such as Reynolds shear stress $\overline{u^{\prime} w^{\prime}}$ ). We use the common notation $U=\bar{u}$ for streamwise velocity. Two-dimensional energy (co)spectral density $E_{\phi \psi}$ is calculated in wall-parallel planes above the riblet tips for streamwise wavelengths $\lambda_{x}$ and spanwise wavelengths $\lambda_{y}$. We collect spectral energy at the positive wavelengths such that their energy integrates to the variance

$$
\int_{0}^{\infty} \int_{0}^{\infty} E_{\phi \psi} \mathrm{d} \lambda_{x} \mathrm{~d} \lambda_{y}=\overline{\phi^{\prime} \psi^{\prime}}(z) .
$$

Accounting for energy from the negative wavelengths at $0<\left(\lambda_{x}, \lambda_{y}\right)<\infty$ is justified because of the symmetry $E_{\phi \psi}\left(\lambda_{x}, \lambda_{y}\right)=E_{\phi \psi}\left(-\lambda_{x},-\lambda_{y}\right)$ and because statistically, $E_{\phi \psi}\left(\lambda_{x}, \lambda_{y}\right) \approx E_{\phi \psi}\left(-\lambda_{x}, \lambda_{y}\right)=$ $E_{\phi \psi}\left(\lambda_{x},-\lambda_{y}\right)$ for real signals $\phi$ and $\psi$. Integrating in only one direction, we define

$$
E_{\phi \psi}^{x}\left(\lambda_{x}, z\right)=\int_{0}^{\infty} E_{\phi \psi} \mathrm{d} \lambda_{y} \quad \text { and } \quad E_{\phi \psi}^{y}\left(\lambda_{y}, z\right)=\int_{0}^{\infty} E_{\phi \psi} \mathrm{d} \lambda_{x} .
$$

When the integration bound is at a finite wavelength, it is important to remember that discrete wavenumbers $k_{i} \in k_{x}$ are centred in their interval, i.e. $E^{i}=\int_{k_{i}-\Delta k_{x} / 2}^{k_{i}+\Delta k_{x} / 2} E^{x}$. 


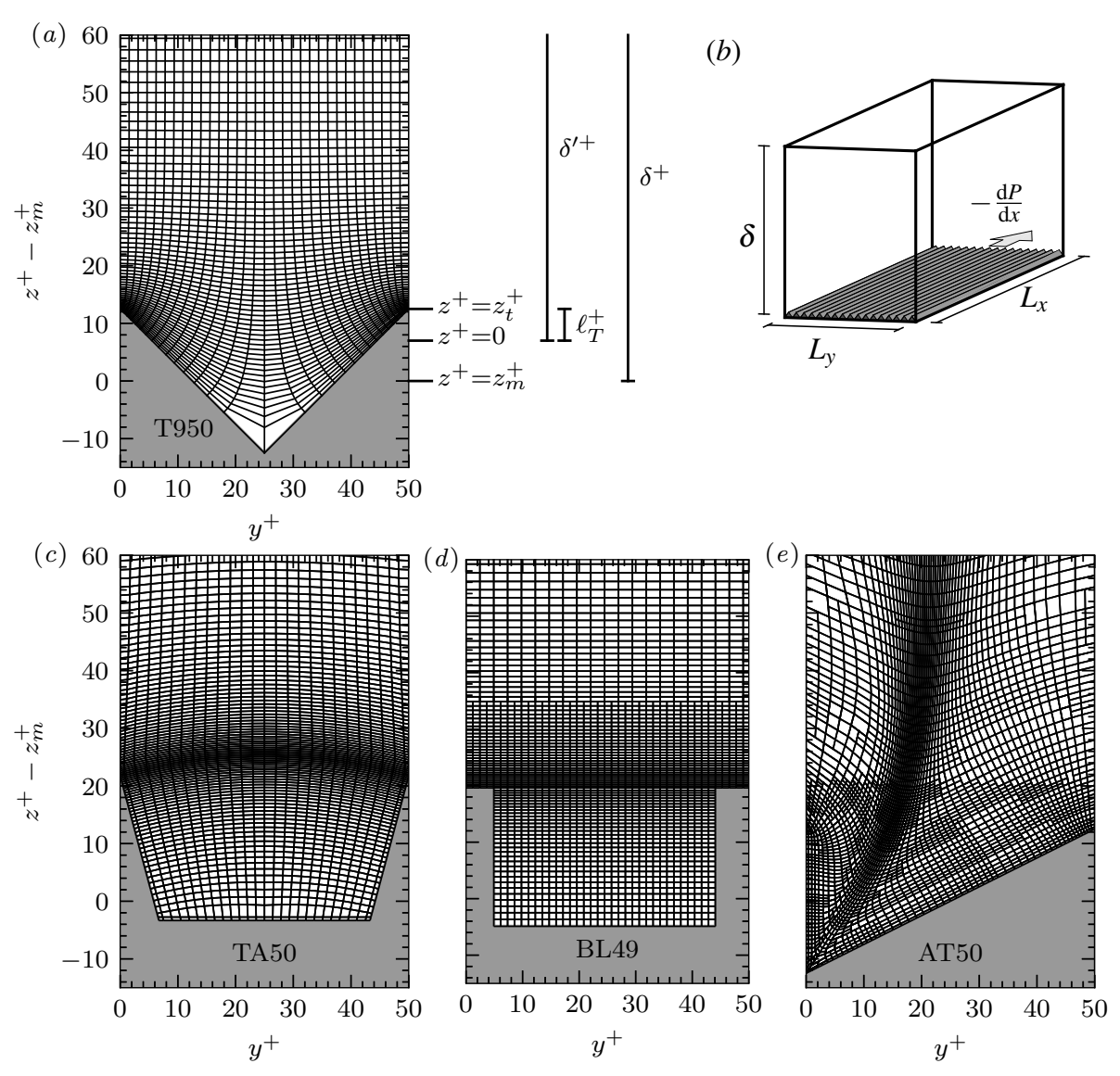

Fig. 1 Computational domain of minimal-span open channels $(b)$ and representative near-wall meshes for the four types of riblets in a cross section spanning one riblet period $(a, c-e)$. The half-channel height $\delta$ is measured from the mean-height $z=z_{m}$ and $\delta^{\prime}$ from the virtual origin $z=0$ with riblet tips at height $z=z_{t}=\ell_{T}$.

\subsection{Origin of the wall-normal coordinate}

We need to define a virtual origin for the wall-normal coordinate in order to measure the drag-change by $\Delta U^{+}$[13], i.e. by the shift in the velocity profile between smooth wall and riblet flow at matched heights. The outer layers of flows over smooth walls and riblets are similar [38] and therefore the latter perceive an equivalent smooth wall at the virtual origin, located $\ell_{T}$ below the riblet crest [27] (figure 1a). We determine $\ell_{T}$ for small riblets near the size of lowest drag by matching profiles of turbulent Reynolds shear stress ${\overline{u^{\prime} w^{\prime}}}^{+}$in the point of largest slope to that of a smooth wall. However, eddies above larger riblets might not perceive a homogeneous boundary that we could use to define the virtual origin [13]. For consistency, we therefore fix $\ell_{T} / k$ for each riblet shape and extrapolate the location of the virtual origin from the small riblets of the same shape. The half-channel height measured from the virtual origin is $\delta^{\prime}<\delta$ (figure $1(a)$ and table 2). 


\subsection{Minimal-span channels}

We employ minimal-span channels for all riblet flows and corresponding smooth-wall references. Minimal-span channels were first used by Jiménez \& Moin [24] and Flores \& Jiménez [10] in numerical experiments on smooth walls and later employed as an economical alternative to costly full-span channel flow DNS for roughness [8]. By definition, the width $L_{y}<\delta$ of minimal-span channels is small enough to constrict large eddies in the flow. This leads to a significantly altered flow field in the outer layer, but below a critical height $z_{c}^{+}$, average velocities and turbulent statistics match experimental results [24].

The study of smooth-wall pipe flow by Chin et al. [6] suggests that a streamwise domain length $L_{x}^{+} \gtrsim 1000$ is necessary to resolve the near-wall streaks and avoid affecting the mean flow. For channel sizes beyond that length, the largest structures are nevertheless not fully resolved and effectively infinitely long in the periodic domain. Such restrictions of the longest structures however do not affect the resolved scales [26]. In the spanwise direction, at least one streak needs to be resolved in a box of width $L_{y}^{+} \gtrsim 100$ for the flow to match experimental data below $z_{c}^{+}$[24]. The critical height above which results are unphysical scales with the domain width as $z_{c}^{+} \approx 0.3 L_{y}^{+}$[10] or less conservatively $z_{c}^{+} \approx 0.4 L_{y}^{+}$[22]. Chung et al. [8] introduce modelled roughness on the wall and also observe $z_{c}^{+} \approx 0.4 L_{y}^{+}$. Roughness only alters the near-wall region of the flow in the roughness sublayer and outer-layer similarity is presumed for flows over different surfaces [38]. In order to capture all roughness effects, we therefore need to choose the channel width such that $z_{c}^{+}$exceeds the height of the roughness sublayer. Consistent with sinusoidal roughness [5], we expect this layer to extend to a height $0.5 s^{+}$, that depends on the lateral riblet spacing $s^{+}$. A systematic analysis of constraints posed by the minimal-span channel is given by MacDonald et al. [30], who vary the streamwise and spanwise extent of numerical domains with modelled roughness to identify and compile limitations for the domain size. For streamwise uniform riblets, these come to

$$
\begin{aligned}
L_{x}^{+} & \gtrsim \max \left(3 L_{y}^{+}, 1000\right), \\
z_{c}^{+}=0.4 L_{y}^{+} & \gtrsim 0.5 s^{+} .
\end{aligned}
$$

Channels of the present study have a width $L_{y}^{+} \approx 250$ at $R e_{\tau}=395$ and $L_{y}^{+}=600$ at $R e_{\tau}=1000$. Therefore, the critical heights are $z_{c}^{+}=0.4 L_{y}^{+} \approx 100$ and $z_{c}^{+}=240$ respectively, both of which are inside the logarithmic layer and above the roughness sublayer $\left(z_{c}^{+}>0.5 s^{+}\right.$ in table 2). Some simulations are repeated in channels with $L_{y}^{+}=150$ and $L_{y}^{+} \approx 450$ (table 2) to analyse effects of the channel width. This enables us to verify that the minimal-span channel constraints found for flow over smooth walls and uniform roughness are valid for the specific case of fully resolved 2D riblets, which differ from traditional roughness in that they do not experience pressure drag. Particularly, since we expect Kelvin-Helmholtz rollers in some of these flows that are not present over many other types of rough surfaces, we verify in $\S 3.2$ that the minimal domains resolve all relevant fluctuations.

Computational grids are stretched in the wall-normal direction using the tanh distribution suggested by Moin \& Kim [31] with smallest spacings near the riblet tips. Crosssections of the meshes close to the riblet surfaces are shown in figure 1 with details given in table 2. Meshes for asymmetric triangular riblets (figure $1 e$ ) are refined using Adapt by Cascade Technologies Inc., which iteratively subdivides cells in different mesh zones (distances from the wall) to meet prescribed requirements for the maximum node spacing in the spanwise and wall-normal direction. Specifically, we set maximum spacings $\Delta y_{m}^{+}=\{1.5,3,4,5\}$ and $\Delta z_{m}^{+}=\{0.9,2,4,6\}$ for heights $z^{+}-z_{t}^{+} \lesssim\{16,40,80\}$ and above respectively. 


\subsection{Runtime and statistical convergence}

We report the statistical uncertainty in $\Delta U^{+}$given by $\zeta^{+}$for all riblet cases in table 2 . In minimal-span channels, $\zeta^{+}$can be estimated based on an eddy-counting approach over the averaging time $L_{t}[30]$

$$
L_{t} \frac{u_{\tau}}{\delta} \approx\left(\frac{91.4}{\zeta^{+} z_{c}^{+}}\right)^{2} \frac{7.5 z_{c}}{L_{x}} \frac{2.5 z_{c}}{L_{y}} \frac{T_{b}^{+}}{L_{z}^{+}}
$$

If $z_{c}^{+}$is in the log-layer of a minimal channel, the bursting period $T_{b}^{+}=6 z_{c}^{+}$[30]. Along with $L_{y}=2.5 z_{c}$, the runtime required to converge statistics in (8) becomes independent of $L_{y}$. Therefore, a further reduction of the spanwise extent of minimal channels (that nevertheless keeps $z_{c}^{+}=0.4 L_{y}^{+}$in the log-layer) reduces the number of cells in the mesh without affecting the runtime, which saves computational costs. In full-span channels, $L_{t}$ depends on both $L_{x}$ and $L_{y}$ and the required runtime is typically much shorter than in minimal-span channels. The larger mesh in $x$ and $y$ however makes the overall computational cost for a full-span channel significantly higher than for a simulation in a minimal channel.

As a measure of statistical convergence, we follow the suggestion by Vinuesa et al. [39] and check (table 2) that the $x-y$-t-averaged total stress above the height of the riblet tips $z_{t}^{+}$ is close to linear

$$
\varepsilon^{\prime+}=\left(\frac{1}{\delta^{\prime+}-z_{t}^{+}} \int_{z_{t}^{+}}^{\delta^{++}} \varepsilon^{+2} \mathrm{~d} z^{+}\right)^{1 / 2}, \quad \text { where } \quad \varepsilon^{+}=\frac{\delta^{\prime+}-z^{+}}{\delta^{+}}+\overline{(u-\bar{u})(w-\bar{w})}+\frac{\mathrm{d} U^{+}}{\mathrm{d} z^{+}} .
$$

\subsection{Mesh convergence study}

The simulation for the narrow version of large blunt triangular riblets $(\mathrm{T} 950 \mathrm{~N})$ is repeated on two coarser meshes as well as a finer mesh (table 2). The number of mesh nodes in each direction are $\left(n_{x}, n_{y}, n_{z}\right)=(257,127,193),(172,97,129),(129,73,97),(87,61,65)$, which means the numbers of nodes spanning one riblet period with $s^{+}=50$ are $n_{s}=43,33,25,21$ (table 2). With fixed domain sizes, the streamwise mesh spacings are $\Delta x^{+} \approx 4,6,8,12$. Our regularly used spacing for all riblet and smooth-wall cases is $\Delta x^{+} \approx 6$ (table 2), which is finer than $\Delta x^{+}=10$ used by Moser et al. [32] at $R e_{\tau}=395$ for a smooth wall DNS with a spectral code. The wall-normal and spanwise grids close to our riblets are likewise finer than for their smooth wall, because the mesh resolution in these directions is dictated by the riblet geometry rather than the viscous length scale. All four cases have a smaller spanwise extent $\left(L_{y}^{+}=150\right)$ than our usual channels $\left(L_{y}^{+} \approx 250\right)$ with minimal-span effects discussed in $\S 3$.

The streamwise spectrum of streamwise velocity fluctuations against distance from the wall shows collapsing contour lines for all four meshes (figure $2 a$ ). Minor differences become visible for Reynolds stresses (figure $2 b$ ) at heights $z^{+}-z_{t}^{+} \gtrsim 20$. Here, the two coarsest meshes have higher fluctuation energy, because the smallest structures are not resolved by the mesh. This directly affects the mean streamwise velocity and therefore profiles of the roughness function $U_{s}^{+}-U^{+}$in figure 2(c), that compares the smooth wall velocity profile (subscript $s$ ) to that of riblets. Below $z_{c}^{+}$, the roughness functions on different meshes are close, but they diverge distinctly towards the top of the domain. Velocities at $z_{c}^{+}$, where we measure the drag-change $\Delta U^{+}=U_{s}^{+}\left(z_{c}^{+}\right)-U^{+}\left(z_{c}^{+}\right)$, are shown in the inset for both the riblet cases (•) and smooth walls with corresponding mesh quality $(\bullet)$. The difference, $\Delta U^{+}$, is almost independent of the mesh quality, provided that the resolution in the streamwise and 

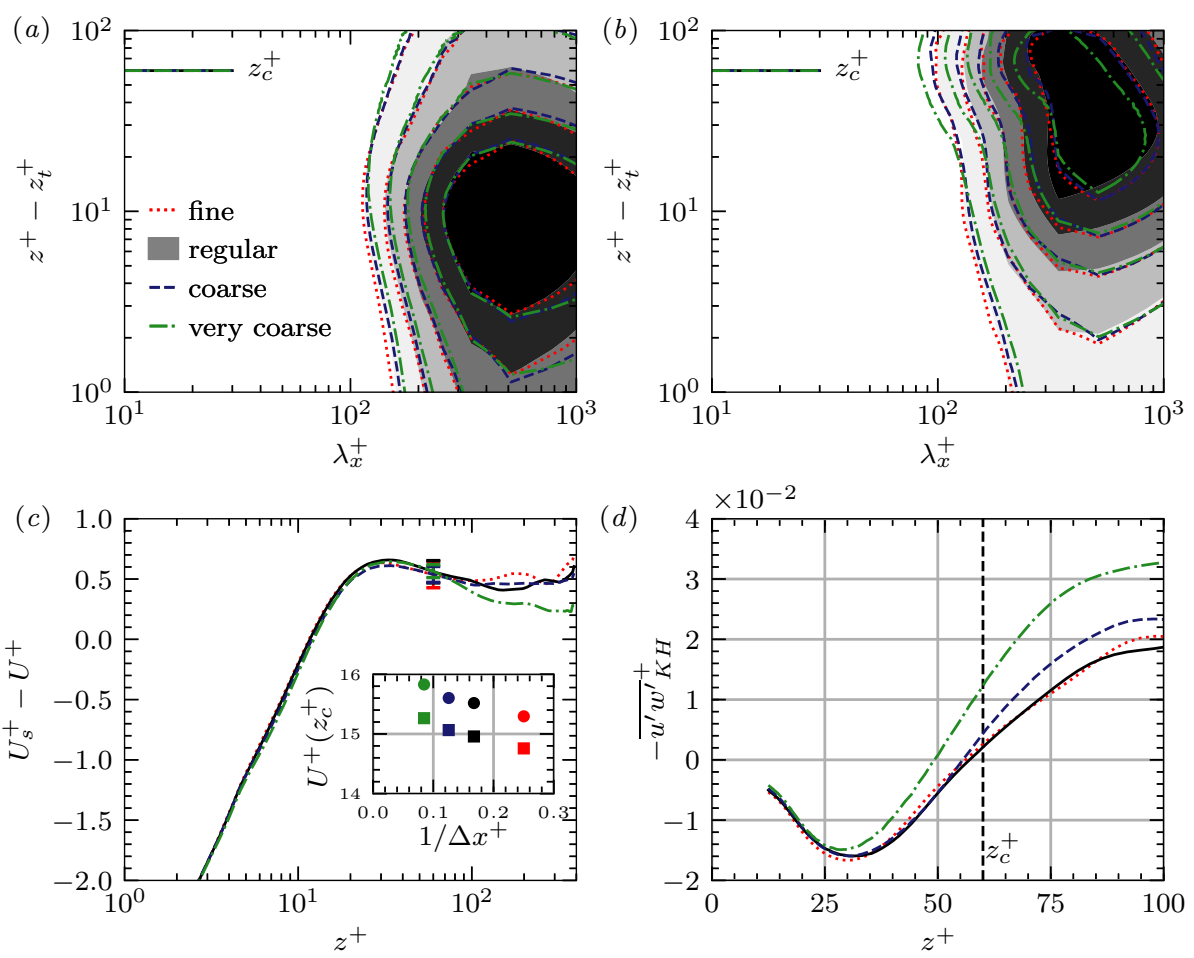

Fig. 2 The solution on coarse meshes deviates, but further refining the computational mesh does not change our results based on these flows in a narrow channel $\left(L_{y}^{+}=150\right)$ with different mesh qualities (T950NF, T950N, T950NC, T950NVC). Premultiplied streamwise spectra with contours from light to dark at $k_{x}^{+} E_{u u}^{x+}=(0.5,0.75,1,1.25,1.5)$ in $(a)$ and $k_{x}^{+} E_{u w}^{x+}=-(0.05,0.1,0.15,0.2,0.25)$ in $(b)$. The roughness function in $(c)$ with error bars for $\Delta U^{+}$measured at $z_{c}^{+}$compares to smooth-wall simulations of matched spanwise extent and mesh resolution (table 2). The inset shows $U_{s}^{+}\left(z_{c}^{+}\right)(\bullet)$ for the smooth wall and $U^{+}\left(z_{c}^{+}\right)(\bullet)$ for riblet flow as a function of the streamwise mesh spacing $\Delta x^{+}$. (d) Reynolds stresses in the spectral region that can be occupied by Kelvin-Helmholtz rollers ([14] and $\S 4.3)$.

wall-normal direction is similar for both flow cases. We therefore maintain $\Delta x^{+} \approx 6$ for all cases and $\Delta z^{+} \approx 0.3$ around the height of the riblet crests and about $\ell_{T}^{+}$above the smooth wall. If we were to use the smooth-wall spanwise resolution for riblets, the geometry would not be resolved properly as we can appreciate by comparing the finest smooth-wall SNF $\left(L_{y}^{+} / n_{y}=2.3, \Delta x^{+} \approx 4\right)$ and coarsest riblet mesh T950NVC $\left(L_{y}^{+} / n_{y}=2.5, \Delta x^{+} \approx 12\right)$. The $\Delta U^{+}$between those mismatched meshes with similar $L_{y}^{+} / n_{y}$ would be close to zero ( - - in the inset of figure $2 c$ ), which is unphysical for riblets with $\ell_{g}^{+}=25$. The average spanwise spacing around riblets is therefore significantly smaller than that of the smooth-wall mesh and $n_{s} \gtrsim 26$ in table 2 .

Reynolds stresses that may be affected by Kelvin-Helmholtz rollers (details in $\S 4.3$ ) are shown in figure $2(d)$. The two coarse meshes have increased energy in this spectral region, even though the considered wavelengths are greater than the mesh spacing $\left(65 \lesssim \lambda_{x}^{+} \lesssim 290\right.$ and $\lambda_{y}^{+} \gtrsim 250$ ). Profiles of Reynolds stresses in that spectral region $-{\overline{u^{\prime} w^{\prime}}}_{K H}^{+}$for the two finer meshes align, even above $z_{c}^{+}$. This gives us confidence that the regular mesh resolution, which we also use for the main cases of this study (those with $L_{y}^{+} \approx 250$ in table 2), is sufficient to capture the effects of Kelvin-Helmholtz rollers on the flow field over riblets. 


\section{The minimal-span channel for flow over riblets}

In this section, we verify that constricting the flow in the spanwise direction unphysically alters the flow in the outer layer, while the near-wall region remains unchanged [30]. Figure 3 shows wall-normal velocity fluctuations 5 viscous units above riblets in channels of three different widths. At this height, the flow fields in all three domains have qualitatively the same streamwise-aligned features, because only flow structures above certain sizes and wallnormal distances are affected by the spanwise constraint. We will quantify these effects in spectral space after first considering implications for the mean streamwise velocity.

\subsection{Mean streamwise velocity in minimal-span channels with riblets}

Constricting the flow in the spanwise direction leads to unphysically high streamwise velocity for heights $z^{+}>z_{c}^{+}=0.4 L_{y}^{+}$in rough-wall channels, because the largest structures that would otherwise carry momentum are not resolved [8]. This estimate for $z_{c}^{+}$agrees well with profiles of mean streamwise velocity shown in figure 4(a) for triangular riblets and smooth walls in numerical domains of three different widths. The viscous-scaled spanwise extents are $L_{y}^{+}=\{150,250,450\}$. Increased velocities above $z_{c}^{+}$are clearly visible for the narrow and medium width channels. The smooth-wall profile from the wide channel with spanwise extent $L_{y}^{+}=450$ is almost identical to that from a full-span channel with $L_{y}^{+}=1241$ except for the very top of the wake region, because $z_{c}^{+}=180$ is fairly close to $\delta^{+}=395$ on a log-scale.
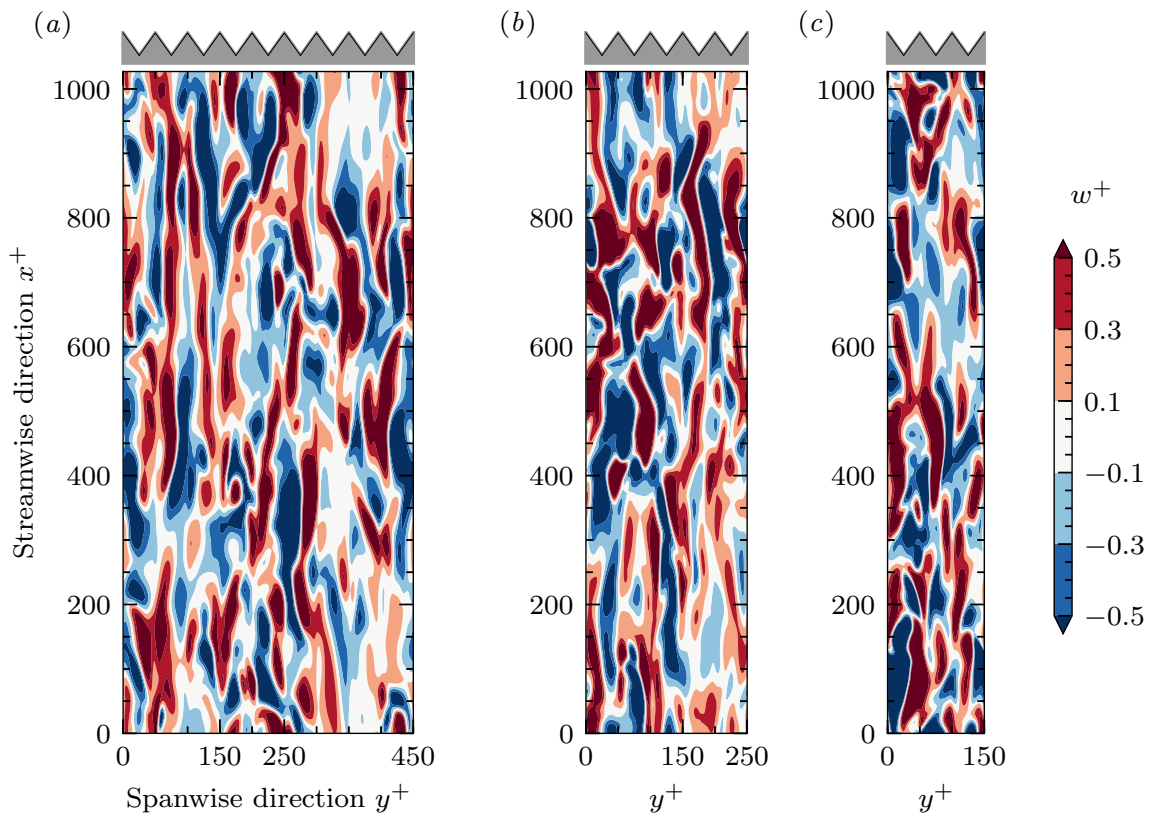

Fig. 3 The flow close to the wall is not affected by the spanwise constraint: instantaneous wall-normal velocity $w^{+}$in a plane 5 viscous units above the crest of triangular riblets with opening angle $\alpha=90^{\circ}$ in channels of different spanwise extent. (a) wide T950W with $L_{y}^{+}=450,(b)$ our regular width T950 with $L_{y}^{+}=250$ and $(c)$ narrow T950N with $L_{y}^{+}=150$. 

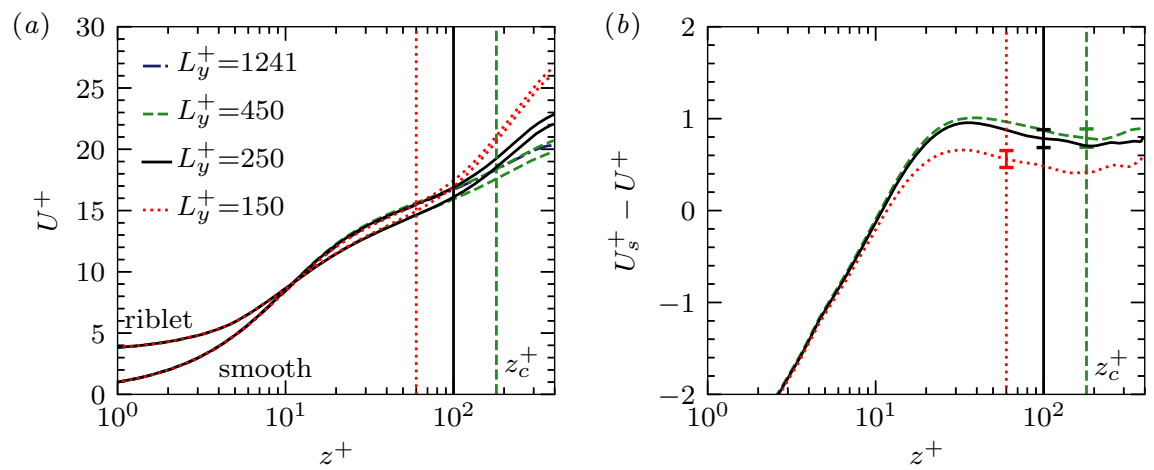

Fig. 4 Minimal channels have unphysically high velocity only above the height $z_{c}^{+}$: $(a)$ velocity profiles of smooth-walls (SW, S, SN) and triangular riblets (T950W, T950, T950N) in domains of three different widths. A profile for a smooth-wall full-span channel (SFull) with $L_{y}^{+}=1241$ is shown additionally for reference. $(b)$ The Hama roughness function $U_{s}^{+}-U^{+}$for the three cases. Vertical lines mark the height $z_{c}^{+}=0.4 L_{y}^{+}$beyond which results are unphysical and error bars mark the statistical uncertainty $\pm \zeta^{+}$as defined in (8).

We calculate the roughness function from the difference between the smooth-wall reference (subscript $s$ ) and the riblet profile at matched spanwise extent $L_{y}^{+}$(figure $4 b$ ). The statistical uncertainty $\zeta^{+}$(found through equation 8 ) is marked by error bars at the height $z_{c}^{+}$, where we measure the drag-change $\Delta U^{+}$. These riblets with groove size $\ell_{g}^{+}=25$ (table 2) are well within the drag-increasing regime as $\Delta U^{+}>0$. For the two widest minimal-span channels, $U_{s}^{+}-U^{+}$collapses within the statistical uncertainty such that the measured drag-change is the same regardless of the domain size. The very narrow channel with $L_{y}^{+}=150$ has a noticeably different roughness function (figure $4 b$ ) and the drag-change $\Delta U^{+}$measured at its $z_{c}^{+}$deviates a bit from the $\Delta U^{+}$found in wider channels (table 2 ). We therefore use $L_{y}^{+} \approx 250$ to study the flow over riblets and thus avoid minimal-span effects on the drag-change.

\subsection{Velocity fluctuations in minimal-span channels with riblets}

Our region of interest for this study is close to the wall, where roughness affects the flow. Kelvin-Helmholtz rollers can appear at heights $\lesssim 20$ viscous units above the riblets [14] and their analysis requires an accurate representation of turbulent flow structures. However, above a certain height in minimal-span channels, the distribution of velocity fluctuations across spectral space is affected by the confined domain. That critical height for fluctuations depends on the size of considered structures as well as $L_{y}^{+}$and it is generally lower than the $z_{c}^{+}$for the mean-flow $[22,30]$.

\subsubsection{Smooth walls and triangular riblets in channels with $L_{y}^{+}=\{150,250,450\}$}

Unphysically amplified velocity fluctuations are evident in the streamwise spectra above $z_{c}^{+}$in our narrow domains with both smooth-walls and riblets (figure $5 a, b$ ). The same amplification is observed by Hwang [22] (their figure 6b) and MacDonald et al. [30] (their figure $4 e-h)$. Closer to the wall however, fluctuations become progressively less dependent on the channel width. 

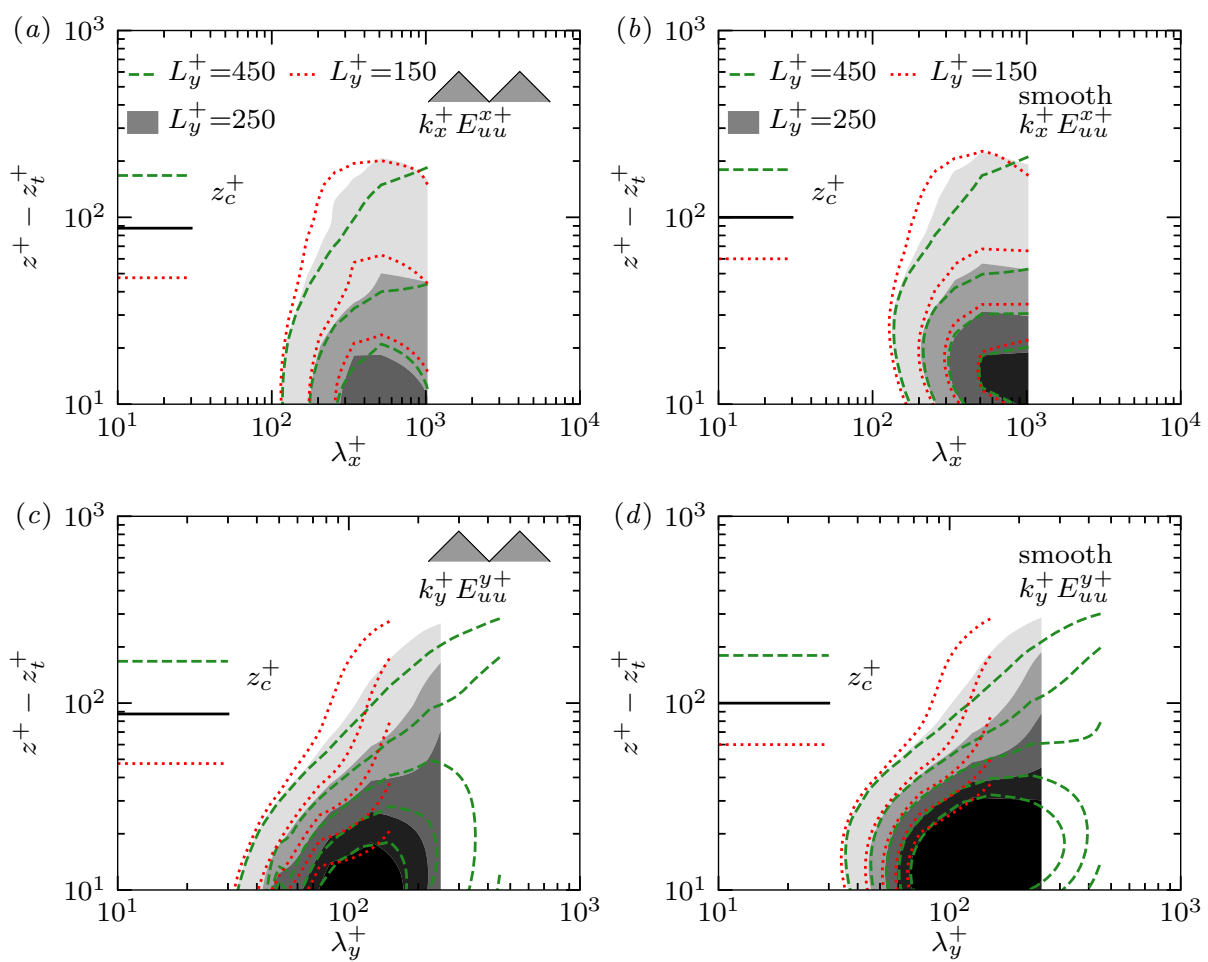

Fig. 5 Velocity fluctuations in minimal channels carry unphysically high energy above the height $z_{c}^{+}$: triangular riblets $\left(\alpha=90^{\circ}\right)$ with three different channel widths (left, T950W, T950, T950N) and corresponding smooth walls (right, $\mathrm{SW}, \mathrm{S}, \mathrm{SN})$. Premultiplied streamwise $(a, b)$ and spanwise $(c, d)$ spectra of streamwise velocity against distance from the riblet crest or wall with contours from light to dark at $k_{x, y}^{+} E_{u u}^{x, y+}=(0.5,1,1.5,2,2.5)$.

Effects of the narrow domain on spanwise spectra are particularly pronounced in figure $5(c, d)$, where wide fluctuations with large $\lambda_{y}^{+}$have increased energy as the domain becomes more narrow. Structures wider than $L_{y}^{+}$appear as spanwise uniform and their energy consequently accumulates in the mode with $\lambda_{y}^{+}=\infty$, which is not visible in these spectrograms. As $L_{y}^{+}$reduces, more fluctuations would be wider than the channel and therefore appear in the infinite wavelength. A side effect of this restriction appears to be an increase of energy in wavelengths just shorter than $L_{y}^{+}$. The width of structures affected by this amplification varies with distance from the wall, as also seen in [30]. Taking the widest channel as a reference, the lowest-energy contour line diverges roughly at $z_{c}^{+}$for the two more narrow channels. Higher-energy contour lines diverge closer to the wall, but also at larger $\lambda_{y}^{+}$. For the medium-width channel, all resolved spanwise wavelengths appear to show unrestricted turbulence below $z^{+}-z_{t}^{+} \approx 30$. For the narrow channel however this is not achieved even at the highest-energy contour level suggesting that energy in the widest structures is overestimated down to some height closer to the riblets or smooth wall respectively.

Figure 6 shows that the region even closer to the wall, only 5 viscous units above the riblets or smooth wall, is not affected by any of our narrow domains. At this height, all contour lines agree across the resolved scales regardless of $L_{y}^{+}$, including for $L_{y}^{+}=150$, because energy at larger wavelengths accumulates at $\lambda_{y}^{+}=\infty$. 

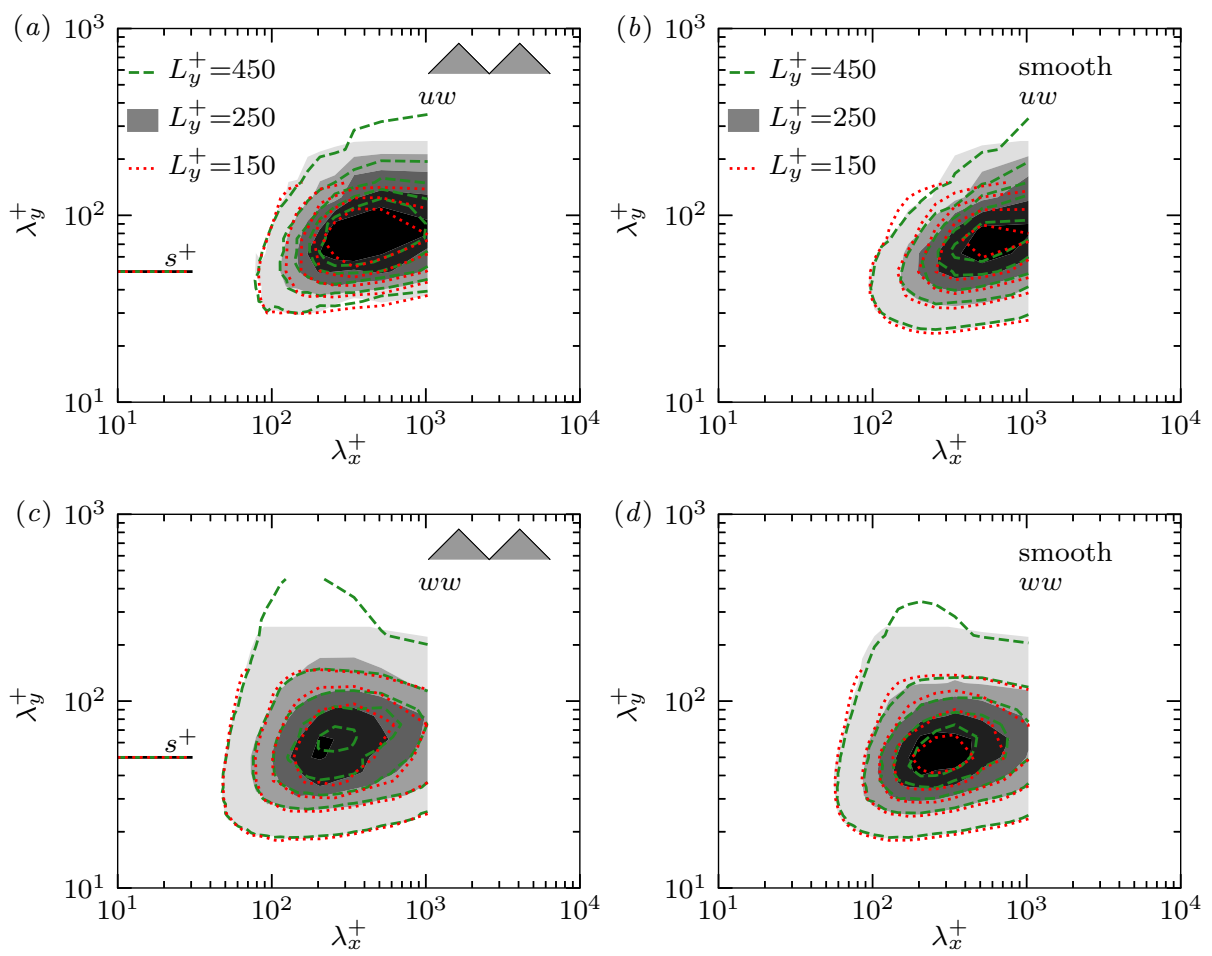

Fig. 6 Velocity fluctuations close to the wall are not affected by the spanwise constraint: triangular riblets $\left(\alpha=90^{\circ}\right)$ with three different channel widths (left, T950W, T950, T950N) and corresponding smooth walls (right, SW, S, SN). Premultiplied 2D spectra in a plane 5 viscous units above the crest with contours at fractions of turbulent fluctuations $k_{x}^{+} k_{y}^{+} E_{\phi \psi}^{+} /\left(\overline{\phi^{\prime} \psi^{\prime}}\right)^{+}=(0.02,0.08,0.14,0.2,0.26)$. In $(a, b)$ for $u w$ and in $(c, d)$ for $w w$.

\subsubsection{Blade riblets in minimal-span and full-span channels}

In figure 7 we further compare spectra of two blade riblet cases in minimal-span channels to similar reference cases from García-Mayoral \& Jiménez [15] in full-span channels to verify that we accurately capture all relevant fluctuations. The reference cases have a spacingto-thickness ratio $s / t=4$ and $R e_{\tau} \approx 550$, while for the blade riblets of this study $s / t=5$ and $\operatorname{Re}_{\tau}=395$, but the riblet sizes are $\ell_{g}^{+} \approx 13$ and $\ell_{g}^{+} \approx 21$ for both data sets. The most notable difference is the channel width. The reference full-span channels are about 9 to 10 times wider in viscous units than our minimal-span channels. The streamwise and spanwise spectra shown against distance from the wall in figure $7(a-d)$ match closely across riblets of a given size, except for the minimal-span channel effects discussed above for the triangular riblets and smooth walls.

Two-dimensional spectra at a height 5 viscous units above the crest, that can be influenced by Kelvin-Helmholtz rollers, are shown in figure $7(e-h)$. The spectra are normalised by their respective turbulent variance at that height and show matching trends regardless of the channel size. Effects of the different blade width are noticeable at this height close to the crest, and presumably explain the small discrepancies between the energy levels (contour lines). However, these misalignments between contour lines for different blade widths are 

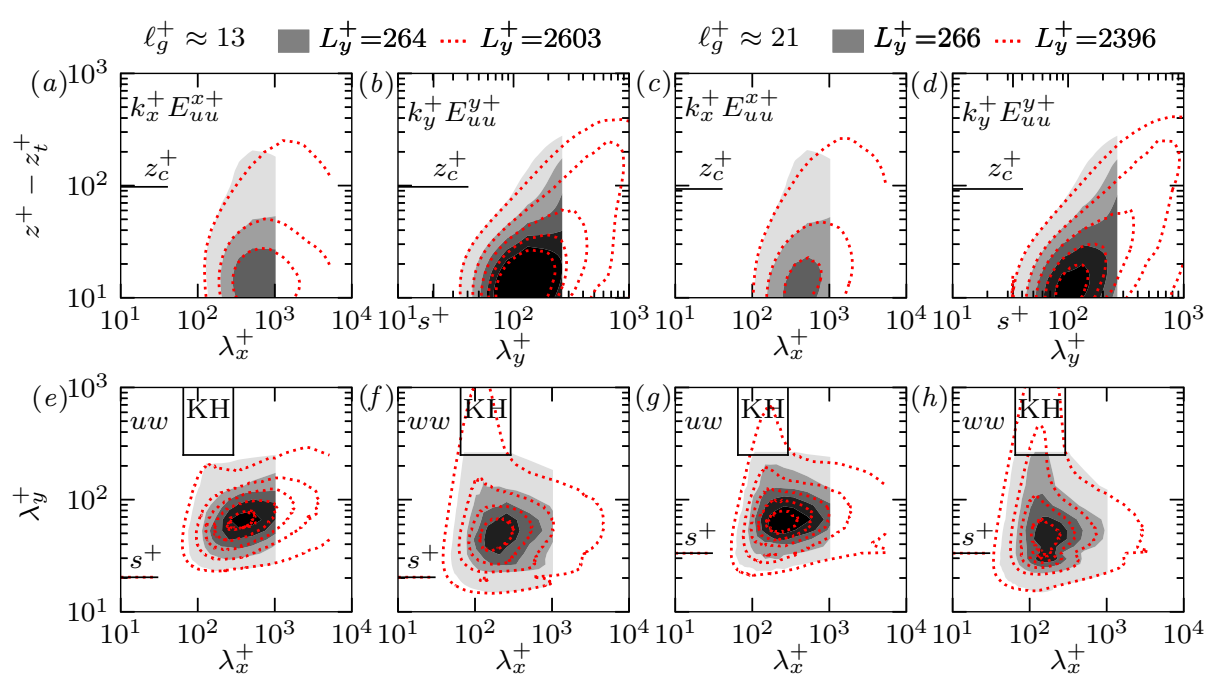

Fig. 7 Comparing velocity spectra for blade riblets in minimal-span channels to reference data in full-span channels. On the left $(a, b, e, f)$ are small riblets with $\ell_{g}^{+} \approx 13$ (BL20 with $L_{y}^{+}=264, R_{\tau}=395, s / t=5$ compared to $13 \mathrm{~L}$ from [15] with $\left.L_{y}^{+}=2603, R e_{\tau} \approx 550, s / t=4\right)$. On the right $(c, d, g, h)$ are large riblets with $\ell_{g}^{+} \approx 21$ (BL34 with $L_{y}^{+}=266, R e_{\tau}=395, s / t=5$ compared to $20 \mathrm{~L}$ from [15] with $L_{y}^{+}=2396, R e_{\tau} \approx 550, s / t=4$ ). The premultiplied streamwise and spanwise spectra of streamwise velocity in $(a-d)$ are shown against distance from the riblet crest with contours from light to dark at $k_{x, y}^{+} E_{u u}^{x, y+}=(0.5,1,1.5,2,2.5)$. The premultiplied 2D spectra of $u w$ in $(e, g)$ and of $w w$ in $(f, h)$ are in a plane 5 viscous units above the crest with contours at fractions of turbulent fluctuations $k_{x}^{+} k_{y}^{+} E_{\phi \psi}^{+} /\left(\overline{\phi^{\prime} \psi^{\prime}}\right)^{+}=(0.02,0.08,0.14,0.2,0.26)$. Boxes near the top delimit the region of Kelvin-Helmholtz rollers ([14] and § 4.3).

small compared to the changes with riblet size (figure $7 e, g$ and figure $7 f, h$ ). The minimalspan channels of the present study (grey contours in figure 7) accurately capture fluctuations of resolved wavelengths close to the wall, as also seen for triangular riblets and smooth walls in figure 6 . The agreement across channels of different width is most readily visible in figure 7( $h$ ) for wall-normal velocity fluctuations above the large riblets, that are known to support the Kelvin-Helmholtz instability [15]. The spectral region of Kelvin-Helmholtz rollers $\left(65 \gtrsim \lambda_{x}^{+} \gtrsim 290\right.$ and $\left.\lambda_{y}^{+} \gtrsim 250\right)$ is framed with a black box near the top of figure $7(e-h)$, but these spectra are restricted to finite wavelength $\lambda_{x}^{+} \lesssim L_{x}^{+}$and $\lambda_{y}^{+} \lesssim L_{y}^{+}$. In the following, we therefore selectively integrate velocity spectra in channels of different width.

\subsubsection{Spectral region of Kelvin-Helmholtz rollers in channels of different widths}

In figure $8(b, d, f, h)$, we show spanwise spectra of Reynolds shear stress integrated in $\lambda_{x}^{+}$only across wavelengths that may be affected by the Kelvin-Helmholtz instability, $65 \gtrsim \lambda_{x}^{+} \gtrsim 290$ [14]. Narrow domains have a low spectral resolution at large $\lambda_{y}^{+}$, but wherever a data point is available, it matches those from wider channels. The sharp triangular riblets with $\alpha=30^{\circ}$ in figure $8(f)$ have more energy in those streamwise wavelengths than the smooth wall in figure $8(b)$ or the blunt triangular riblets with $\alpha=90^{\circ}$ in figure $8(d)$. Nevertheless, for all of them, the energy at large $\lambda_{y}^{+}$matches closely between channels of different width. In figure $8(h)$ we further compare the blade riblets to slightly thicker blades with almost matching $\ell_{g}^{+}$in exceptionally wide full-span channels by García-Mayoral \& Jiménez [15] and also observe close agreement of the energy across all resolved wavelengths. 

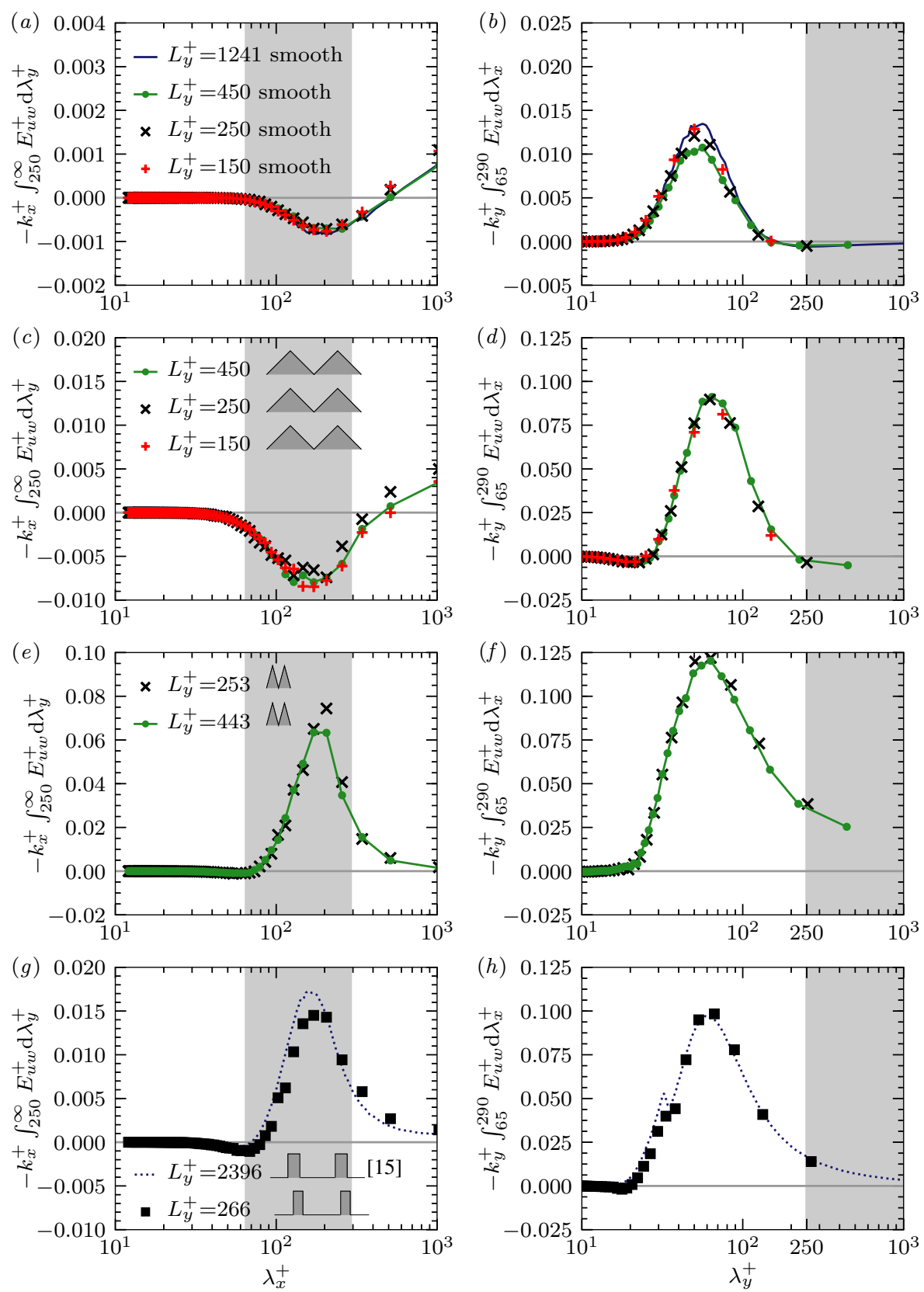

Fig. 8 Minimal channels capture the energy in the spectral region of Kelvin-Helmholtz rollers: premultiplied streamwise (left) and spanwise (right) spectra of Reynolds shear stress integrated only over the shaded wavelengths that are affected by the Kelvin-Helmholtz instability $65 \lesssim \lambda_{x}^{+} \lesssim 290$ and $\lambda_{y}^{+} \gtrsim 250$ ([14] and $\S 4.3$ ). In a plane 5 viscous units above smooth walls $(a, b)$, above the crest of triangular riblets T950W, T950, T950N $\left(\alpha=90^{\circ}, \ell_{g}^{+}=25.0\right.$ in $\left.c, d\right)$, triangular riblets T321W, T321 $\left(\alpha=30^{\circ}, \ell_{g}^{+}=20.4\right.$ in $\left.e, f\right)$ and blade riblets 20L $\left(s / t=4, \ell_{g}^{+}=20.4\right)$ in a full-span channel [15] and BL33 $\left(s / t=5, \ell_{g}^{+}=21.1\right)$ in our minimal-span channel $(g, h)$. 
The spanwise extent of Kelvin-Helmholtz rollers is up to $1000-1500$ viscous units in the flow over blade riblets [15], which means a large portion of their energy accumulates in the spanwise-infinite wavelength of our simulations, which is not visible in the premultiplied spectra of figure $8(b, d, f, h)$. In order to visualise energy in modes with $\lambda_{y}^{+} \gtrsim 250$ including $\lambda_{y}^{+}=\infty$, we integrate cospectra of Reynolds shear stress for the different channels across $\lambda_{y}^{+} \gtrsim 250$ in figure $8(a, c, e, g)$. Smooth wall flow at $z^{+}=5$ has very little energy in these large spanwise wavelengths, but the spectrum is the same for all four channel widths. In the case with comparatively high energy at these large spanwise wavelengths in figure 8(e), the spectrum likewise matches within the statistical uncertainty for both domain widths. Even the narrow domains with $L_{y}^{+}=150$ in figure $8(a, c)$, that end short of the integration bound, have the same spectrum, because the energy is contained in the spanwise-infinite wavelength. (The integration in discrete wavenumber space covers all of mode 0 with $\lambda_{y}^{+}=\infty$ and $10 \%$ of mode 1 with $\lambda_{y}^{+}=150$.) In figure $8(g)$, we integrate the spectrum for our blade riblets over only two spanwise modes $\left(L_{y}^{+}=266\right)$ and the spectrum of the reference case [15] with a similar geometry over 11 modes $\left(L_{y}^{+}=2396\right)$, but the resulting streamwise spectra for large spanwise wavelengths nevertheless match closely.

In summary, we observe in figures 4 and 5 that if $L_{y}^{+} \gtrsim 250$, turbulence in roughly the first 30 viscous units above the riblet crest is not affected by the channel width and both the mean and the turbulent flow that we simulate in that region (including at large $\lambda_{y}^{+}$) reflect the true physics. Furthermore, figures 6 and 8 illustrate that 5 viscous units above the wall or riblet crest, Reynolds stresses and wall-normal velocity in minimal-span channels with $L_{y}^{+} \gtrsim 150$ closely match those in wider domains. Energy at wavelengths $\lambda_{y}^{+}>L_{y}^{+}$accumulates at the infinite wavelength such that fluctuations of large structures are accounted for even in narrow domains (figure 8). Our minimal-span channels with $L_{y}^{+} \approx 250$ (table 2) therefore allow us to investigate fluctuations due to Kelvin-Helmholtz rollers. Even more narrow domains (e.g. with $L_{y}^{+}=150$ ) also accurately capture energy in wavelengths that are affected by the KelvinHelmholtz instability (figure 8), but the separation from other near-wall turbulence is cleaner if $L_{y}^{+} \gtrsim 250$ as we will demonstrate in $\S 4.3$.

\section{The Kelvin-Helmholtz instability above riblets}

\subsection{Roughness function of riblets}

Our riblet DNS data set spans six shapes and various viscous-scaled sizes, which means that drag characteristics vary as shown in figure 9 , where we compare the streamwise velocity to smooth-wall flow in channels of matched width, $\Delta U^{+}=U_{s}^{+}\left(z_{c}^{+}\right)-U^{+}\left(z_{c}^{+}\right)$. Measures of the drag-change $\Delta U^{+}$by blade riblets ( $\square$ ) are slightly lower than for the reference data of thicker blades ( $\square$ ) [15], which is consistent with Stokes flow that predicts the lowest $\Delta U^{+}$ for infinitely thin blades [28]. We further converted various reference data from DR to $\Delta U^{+}$ for comparison. The blunt trapezoidal riblets from Gatti et al. [16] with $\alpha=53.5^{\circ}$ ( $\star$ ) have a higher drag at matched $\ell_{g}^{+}$than our trapezoidal riblets with $\alpha=30^{\circ}\left(\hbar^{\dagger}\right)$. Around the size of optimum drag reduction at $\ell_{g}^{+} \approx 11$, drag curves of various riblet shapes scale with $\ell_{g}^{+}$ [14]. Nevertheless, our triangular riblets $(\nabla, \varangle)$ have a lower minimum drag than measured experimentally by Bechert et al. [3] $(\nabla, \triangleleft)$. The trapezoidal riblets show the same trend of lower drag in numerical compared to experimental studies: our DNS ( $\downarrow$ ) and the wallresolved large eddy simulations by Bannier et al. [1] ( $\lesssim$ ) of trapezoidal riblets with $\alpha=30^{\circ}$ have a lower drag at the optimum than the experimental data from Gatti et al. [16] ( $\star$ ) for trapezoidal riblets with $\alpha=53.5^{\circ}$. The lower drag of the sharper trapezoidal riblets is only 


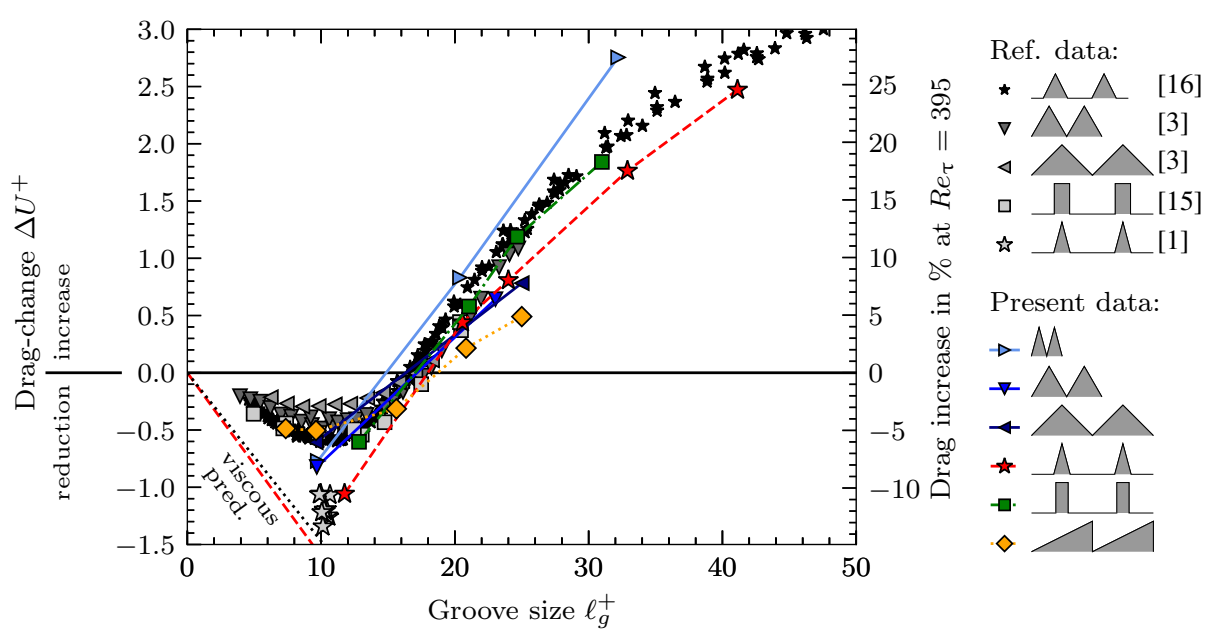

Fig. 9 Drag curves for different riblet shapes with experimental $(\star$ [16], $\nabla$ [3], $\triangleleft$ [3]) and numerical (DNS: $\square$ [15], LES: $\lesssim$ [1]) reference data. Experimental channel flow data are converted from $\Delta \tau_{w} / \tau_{w, \text { smooth }}$ to $\Delta U^{+}=-\operatorname{DR}\left(\left(2 c_{f, \text { smooth }}\right)^{-1 / 2}+1 /(2 \kappa)\right)$ with $c_{f}=2 / U_{\text {bulk }}^{+2}$ at matched $R e_{\text {bulk }}$ [3]. The term $1 /(2 \kappa)$ is omitted for data from [3] as suggested in [12]. The boundary layer LES data are converted to $\Delta U^{+}=-\mathrm{DR}\left(2 C_{f, \text { smooth }}\right)^{-1 / 2}$ with $C_{f}=2 / U_{\delta}^{+2}$ at matched $U_{\delta}^{+}$[37]. Direct measurements of $\Delta U^{+}$for the present data are shown by coloured symbols with lines. The straight lines starting at $\ell_{g}^{+}=0$ indicate the viscous prediction of $\Delta U^{+}$calculated from Stokes flow as per [28], for trapezoidal riblets with $\alpha=53.5^{\circ}(\cdots \cdots \cdot$, as [16]) and $\alpha=30^{\circ}$ (----, as [1] and our data).

partially explained by Stokes flow [28], which predicts a small reduction of $\Delta U^{+}$when the tip angle reduces from $\alpha=53.5^{\circ}$ to $\alpha=30^{\circ}$ (straight lines starting at $\ell_{g}^{+}=0$ in figure 9).

García-Mayoral \& Jiménez [14] demonstrated for blade riblets, that Kelvin-Helmholtz rollers appear in the flow $\lesssim 20$ viscous units above the crest for groove sizes $\ell_{g}^{+} \gtrsim 11$, i.e. for larger than optimal riblets. Fourteen of our cases have a size above that threshold in order to analyse the effects of Kelvin-Helmholtz rollers on the flow field and therefore on the drag characteristics of different riblet geometries.

\subsection{Visualising Kelvin-Helmholtz rollers}

We use the scalar $\theta$, that represents temperature, to visualise perturbations in the fluid. Fluctuations $\theta^{\prime+}$ in a plane 5 viscous units above a smooth wall or small riblets (figure 10, left) show long, nominally streamwise aligned, streaks of positive or negative fluctuations. For some of the larger, drag-increasing riblets however spanwise coherent patches dominate the flow field at that height (figure 10, right). This is most prominently visible in figure $10(c)$ for the triangular riblets with $\alpha=30^{\circ}$, where it suggests the presence of spanwise-coherent Kelvin-Helmholtz rollers. The flow field of the blunt triangular riblets with $\alpha=90^{\circ}$ in figure $10(\mathrm{~g})$ and the asymmetric riblets in figure $10(\mathrm{~m})$ looks qualitatively similar to that of the corresponding small riblets (figure $10(f, l)$ respectively), where no significant spanwise coherence is visible at this instance. These instantaneous flow visualisations therefore suggest that the strength of Kelvin-Helmholtz rollers depends on the riblet shape.

Figure 11 shows isosurfaces of the passive scalar to visualize spanwise coherence in a volume $z^{+}-z_{t}^{+}<20$, highlighting the striking dependence of the Kelvin-Helmholtz instabil- 

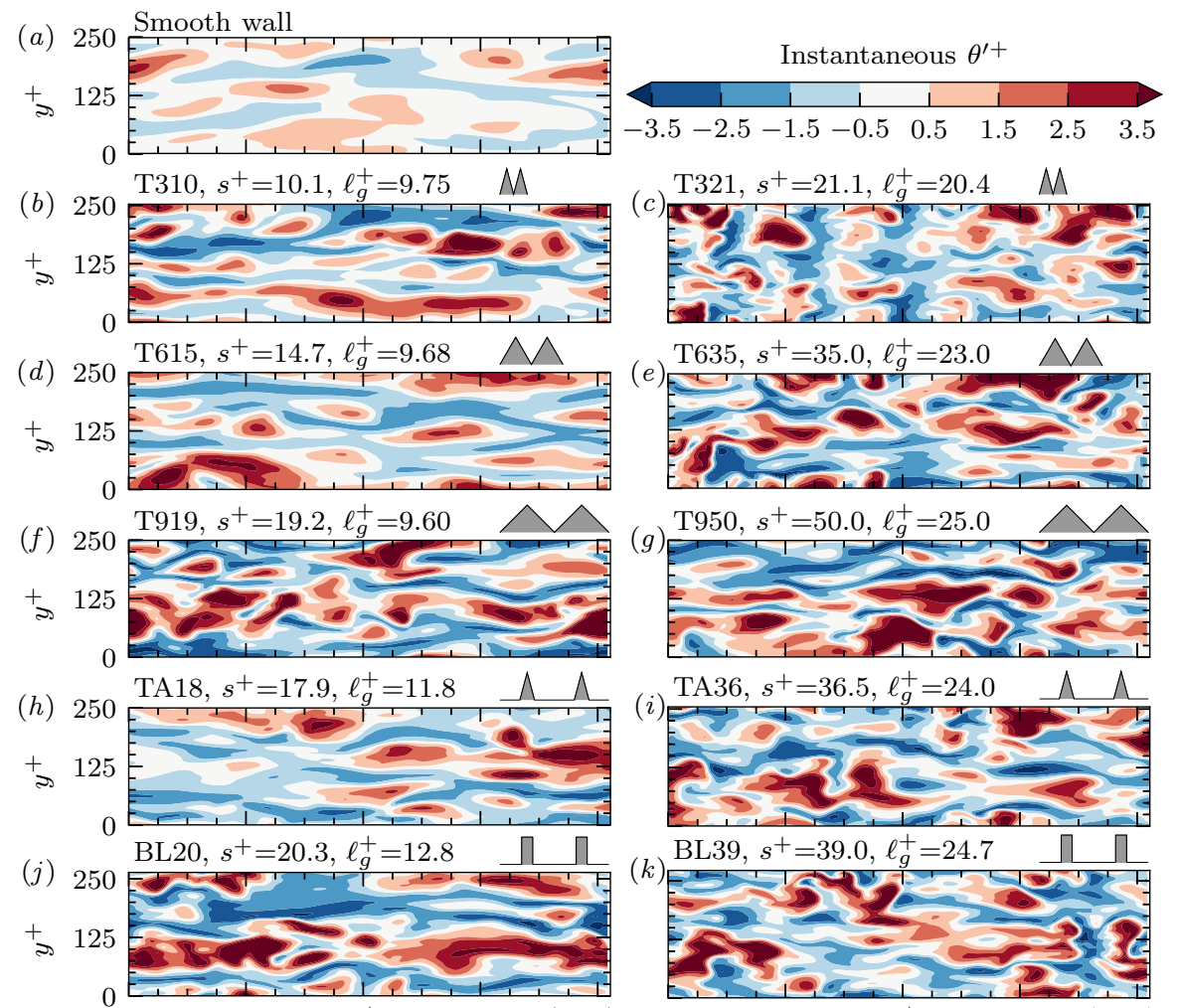

$(k)$
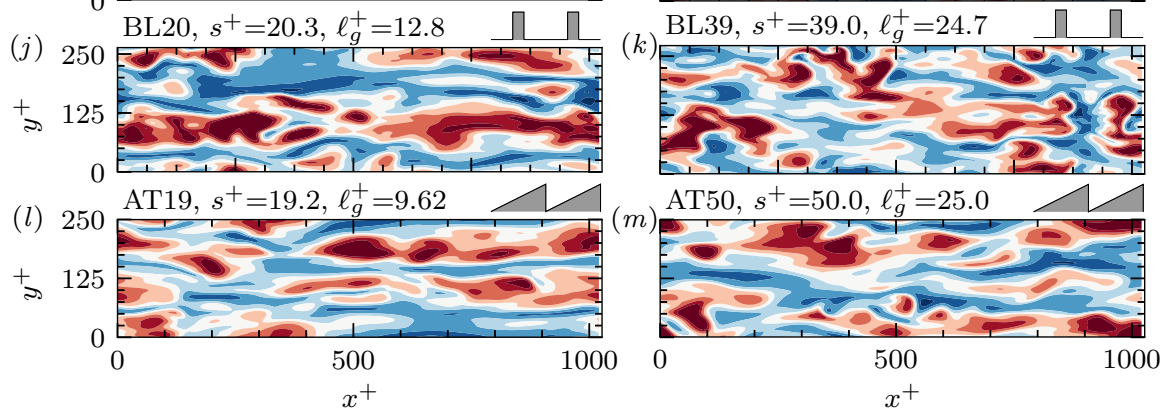

Fig. 10 Spanwise coherence due to Kelvin-Helmholtz rollers is visible for some of the large riblets: fluctuations of the passive scalar (temperature) $\theta^{\prime+}$ in the wall-parallel plane 5 viscous units above a smooth wall $(a)$ or riblet crest $(b-m)$. Riblet cases on the left are close to their drag-reducing optimum size, while those on the right side are large enough to be in the drag increasing regime (figure 9).

ity on the riblet cross-section. The two riblet geometries are the same as in figure $10(c, g)$, except in wider domains with $L_{y}^{+} \approx 450$. In combination with regions of negative streamwise velocity in the groove of the large triangular riblets with $\alpha=30^{\circ}$ (blue in figure $11 a$ ), wavelike structures become visible in the flow field, which match the description of a KelvinHelmholtz instability (roughly spanwise coherent and with a streamwise spacing $\lambda_{x}^{+} \approx 200$ ). Kelvin-Helmholtz rollers are absent above the blunt riblets with $\alpha=90^{\circ}$ in figure $11(b)$, even though the riblet size $\ell_{g}^{+}$is similar for both cases and the domain size and $R e_{\tau}$ are matched.

In order to quantify the intensity of the Kelvin-Helmholtz instability and its relevance in the flow field, we now apply a spectral filter to time-averaged fluctuating quantities. 


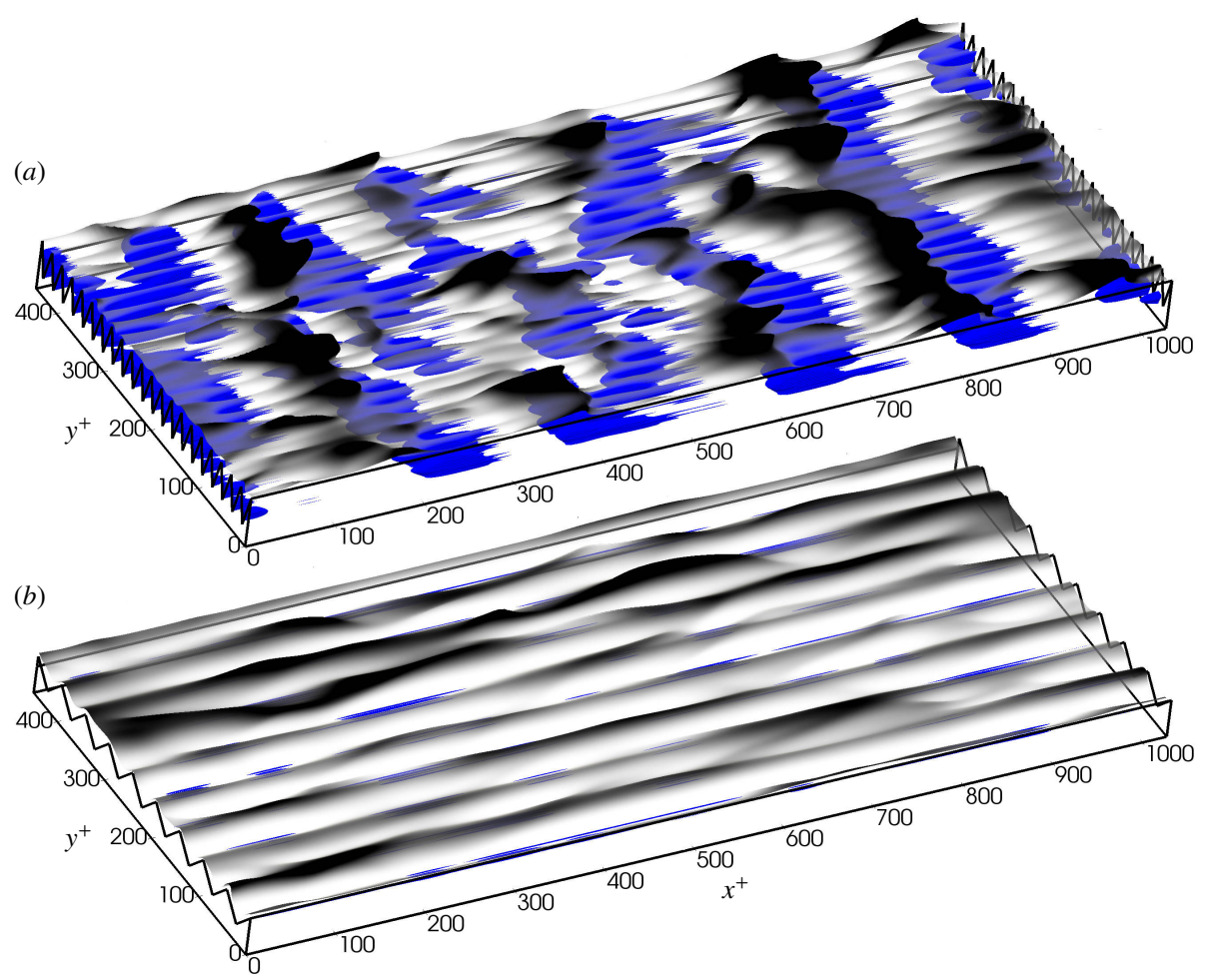

Fig. 11 Flow visualisation for two drag-increasing riblets of similar size $\ell_{g}^{+}$but different shape. KelvinHelmholtz rollers develop over the triangular riblets with tip angle $\alpha=30^{\circ}$ ( $a$ : T321W with $\ell_{g}^{+}=20.4$ ), but not over the riblets with $\alpha=90^{\circ}\left(b\right.$ : T950W with $\ell_{g}^{+}=25$ ). Both channels have a spanwise extent $L_{y}^{+} \approx 450$. The mean flow is aligned with the riblets from left to right. Regions of negative streamwise velocity (reversed flow) inside the groove are shown in blue. An isosurface of the passive scalar $\theta^{+}=3.5$ above the riblet crest is coloured by its height from transparent to black.

\subsection{Identifying Kelvin-Helmholtz rollers in spectral space of minimal channels}

For sufficiently large blade riblets, spanwise coherent rollers related to a Kelvin-Helmholtz instability form just above the riblet crest [14]. These structures can be identified in spectral space, where they alter velocity fluctuations in the region roughly delimited by $65 \lesssim \lambda_{x}^{+} \lesssim 290$ and $\lambda_{y}^{+} \gtrsim 130$ according to García-Mayoral \& Jiménez [14].

\subsubsection{Signature of Kelvin-Helmholtz rollers in $2 D$ spectra}

In figure 12 , we show premultiplied 2D spectra of various flow quantities normalised by their turbulent variance at a height 5 viscous units above drag-increasing triangular riblets with $\alpha=30^{\circ}$. This normalisation visualises how these riblets change the distribution of energy across both spectral dimensions relative to the smooth wall (----). Energy in the spectral region associated with the instability (box at the top in figure 12) is altered in spectra of $u$, $v, w$ and $\theta$. Pressure fluctuations are shifted to slightly lower $\lambda_{x}^{+}$, but contour lines mostly retain the shape of smooth-wall flow. Wall-normal velocity and temperature fluctuations in particular are strongly affected with a distinct peak in the spectral region of the instability. 

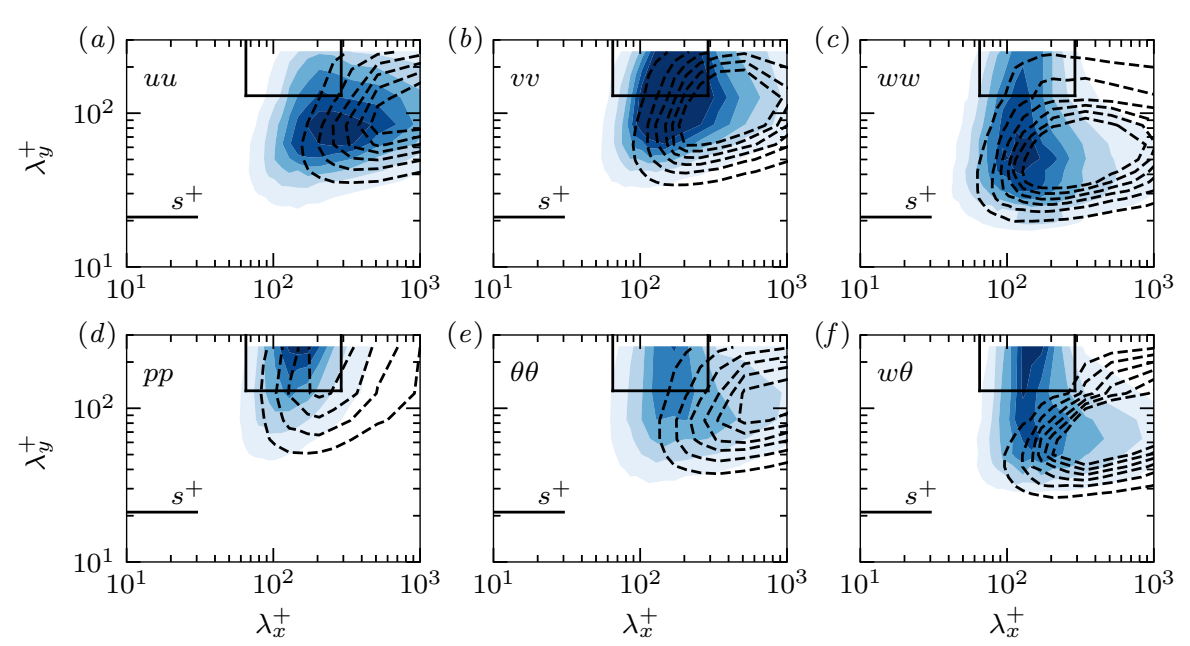

Fig. 12 Kelvin-Helmholtz rollers changing the distribution of energy in premultiplied 2D spectra in a plane 5 viscous units above the crest of drag increasing sharp triangular riblets T321 (contours) compared to smooth-wall flow (----). Contours are from light to dark at fractions of turbulent fluctuations $k_{x}^{+} k_{y}^{+} E_{\phi \psi}^{+} /\left(\overline{\phi^{\prime} \psi^{\prime}}\right)^{+}=(0.03,0.06,0.09,0.12,0.15,0.18)$. Boxes near the top delimit the region of KelvinHelmholtz rollers $\left(65<\lambda_{x}^{+}<290, \lambda_{y}^{+}>130\right)$ according to [14].

The effect of Kelvin-Helmholtz rollers on fluctuations in the temperature field has implications for heat transfer of the surface. The temperature is modelled as a passive scalar that cannot affect Kelvin-Helmholtz rollers. However, the Kelvin-Helmholtz instability can enhance the transport of the passive scalar into the wall, akin to forced convection of heat. The differences between figure 12(a) and figure 12(e) indicate that Kelvin-Helmholtz rollers affect heat transfer differently from momentum transfer into the wall. The reversed flow regions seen in figure 11 $(a)$ have negative wall-shear stress and positive heat transfer into the wall, affecting the overall ratio of heat transfer to momentum transfer and thus breaking the similarity implied by the Reynolds analogy.

\subsubsection{Signature of Kelvin-Helmholtz rollers in $2 D$ shear stress cospectra}

We further consider the spectrum of Reynolds shear stress, because this is the component of the Reynolds stress tensor that appears in the mean streamwise momentum equation, where it directly relates to the drag variation $[11,14,29]$. Figures $13(a, b)$ show normalised 2D spectra of Reynolds shear stress in a plane 5 viscous units above the crest of triangular riblets with $\alpha=30^{\circ}$. For the small drag-reducing riblets (T310), contours are similar to those above a smooth wall (----). An impermeable smooth wall does not provide the conditions for the Kelvin-Helmholtz instability to develop and the same is true for small riblets, typically below the size of optimum drag, i.e. $\ell_{g}^{+} \lesssim 11$ [14]. Therefore, the spectrum of Reynolds shear stress contains negligible energy in the associated spectral region.

The spectral region of Kelvin-Helmholtz rollers for the drag-increasing riblets of the same shape (T321 in figure 13b) features a strong peak and the distribution of Reynolds shear stress across the 2D spectrum is distinctly different from that over a smooth wall, as discussed for figure 12. The bulk of the energy over smooth walls is distributed around wavelengths close to $\lambda_{x}^{+} \approx 1000$ (e.g. [21]) with high energy down to $\lambda_{x}^{+} \approx 300$ based on 

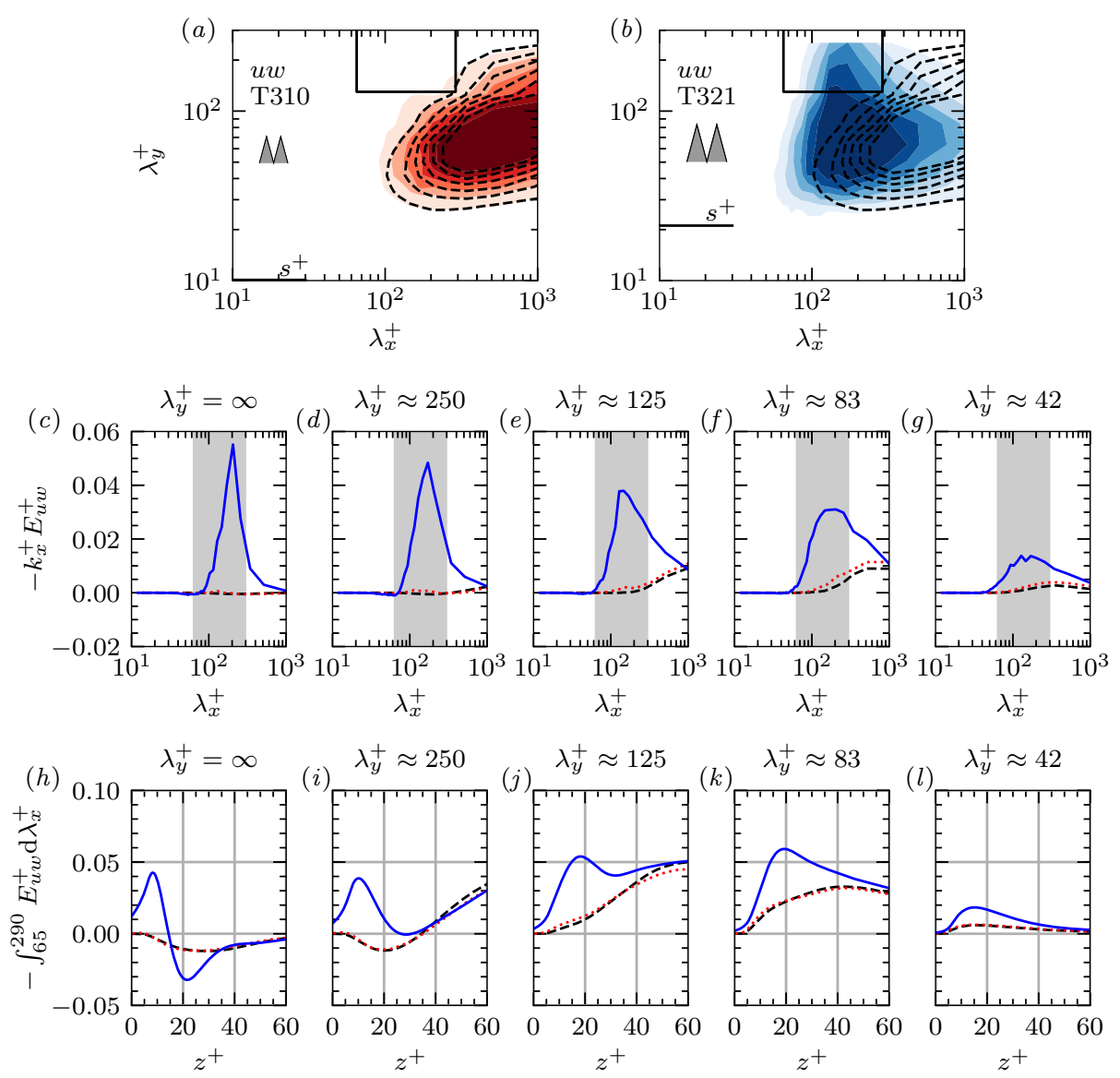

Fig. 13 Determining which spanwise wavelengths are affected by Kelvin-Helmholtz rollers using drag reducing (T310) and drag increasing (T321) sharp triangular riblets with tip angle $\alpha=30^{\circ}$ and a smooth wall. $(a, b)$ Premultiplied 2D cospectra of Reynolds shear stress in a plane at 5 viscous units above the crest. Contours are from light to dark at fractions of turbulent fluctuations $k_{x}^{+} k_{y}^{+} E_{u w}^{+} /\left(\overline{u^{\prime} w^{\prime}}\right)^{+}=(0.03,0.06,0.09,0.12,0.15,0.18)$, filled for riblets and lines for the smooth wall. Open boxes near the top delimit the region of Kelvin-Helmholtz rollers $\left(65<\lambda_{x}^{+}<290, \lambda_{y}^{+}>130\right)$ according to [14]. $(c-g)$ Premultiplied 1D cospectra of Reynolds shear stress at different spanwise wavelengths (modes $0,1,2,3,6)$ for a smooth wall (----), a drag reducing riblet $(\mathrm{T} 310, \cdots \cdots)$ and a drag increasing riblet (T321, $-)$ with matched domain size. (h-l) Profiles of Reynolds shear stress at different spanwise wavelengths after integrating across the $\lambda_{x}^{+}$that are framed in $(a),(b)$ and shaded in $(c-g)$.

figure 3(c) in [23] or the contour lines in our figure 13(b). However, for the large riblets with $\alpha=30^{\circ}$ in figure $13(b)$, the strong peak is instead at $\lambda_{x}^{+} \approx 200$ and the relative influence of large streamwise structures on Reynolds stress at this particular height is reduced significantly compared to smooth-wall flow.

\subsubsection{D shear stress cospectra at individual spanwise wavelengths}

The aim now is to isolate the effect of the Kelvin-Helmholtz instability by integrating fluctuations only over the wavelengths affected by Kelvin-Helmholtz rollers. As visible in the 
2D spectrum of figure 13(b), the peak associated with the instability is an extension of the main peak of near-wall turbulence at lower spanwise wavelengths and not entirely separated from it, at least along the $\lambda_{y}^{+}$dimension.

In an effort to judge how far the influence of Kelvin-Helmholtz rollers extends, streamwise spectra at large spanwise wavelengths are shown in figure 13(c-g), including at $\lambda_{y}^{+}=$ $\infty$, which is not visible in the premultiplied 2D spectra (figure 13a,b). The streamwise region that García-Mayoral \& Jiménez [14] identified for the instability (shaded in figure 13c-g) contains the peak in the spectrum (-). Since we are employing minimal-span channels with $L_{y}^{+} \approx 250$, the spanwise-infinite wavelength (figure 13c) contains a large portion of the energy. In fact, the peak associated with Kelvin-Helmholtz rollers at the infinite wavelength is stronger than at any other wavelength resolved in this channel, which underlines the spanwise two-dimensional nature of the Kelvin-Helmholtz rollers. For $\lambda_{y}^{+}=L_{y}^{+} \approx 250$ in figure $13(d)$, the distribution of energy across $\lambda_{x}^{+}$is similar to the one for the spanwiseinfinite wavelength in figure 13(c), although the position of the peak reduces from $\lambda_{x}^{+} \approx 205$ at $\lambda_{y}^{+}=\infty$ to $\lambda_{x}^{+} \approx 170$. Both peaks are inside the shaded region of $\lambda_{x}^{+}$, but for smaller wavelengths (figure $13 e-g$ ), the energy peak is reducing in strength and broadening as fluctuations from the near-wall-cycle are affecting the spectrum (also visible in figure $13 b$ ).

In contrast to the large riblets $(-)$, the curves for the small drag-reducing riblets $(\cdots \cdots .$. in figure $13(c-g)$ are almost indistinguishable from those of smooth-wall flow (----), because the contours in figure 13(a) do not extend into the spectral region of Kelvin-Helmholtz rollers for either flow. We interpret smooth-wall like flow over riblets in the spectral region of the instability as Kelvin-Helmholtz rollers not being present, which we expect for riblets with $\ell_{g}^{+} \lesssim 11$ that lack wall-normal permeability [14].

\subsubsection{Profiles of shear stress contributed by individual spanwise wavelengths}

This analysis is so far limited to one representative distance from the wall, 5 viscous units above the crest. However, we can expect Kelvin-Helmholtz rollers to be three-dimensional structures and to account for the $z$-dependence, we integrate Reynolds stresses at every height across the range of streamwise wavelengths $65 \lesssim \lambda_{x}^{+} \lesssim 290$ that are affected by the instability. For now, we integrate separately at every spanwise wavelength to obtain profiles of Reynolds stresses due to Kelvin-Helmholtz rollers shown in figure 13( $h-l)$ that depend on $\lambda_{y}^{+}$. The drag reducing riblet case (.....) has profiles that are very close to that of the smooth wall (----) at all spanwise wavelengths. For the drag increasing case (-), the effect of Kelvin-Helmholtz structures is evident. In agreement with the description by García-Mayoral \& Jiménez [14] and visible in figure 13( $h, i)$, Kelvin-Helmholtz rollers increase energy in large spanwise wavelengths for roughly the first 20 viscous units above the riblet crest. At $\lambda_{y}^{+} \approx 125$ (figure $13 j$ ), the peak is lower and farther from the wall as structures from the near wall cycle are beginning to affect the profile. Particularly for the graphs in figure $13(k, l)$ at $\lambda_{y}^{+}<100$, the profile no longer captures energy associated exclusively with Kelvin-Helmholtz rollers, but mainly with the near-wall cycle. This is more readily visible in the $2 \mathrm{D}$ spectrograms of figure $13(b)$.

\subsubsection{Summary of scales affected by Kelvin-Helmholtz rollers}

The above spectral analysis of the flow over sharp triangular riblets demonstrates that the lower bound for the spanwise extent of Kelvin-Helmholtz rollers should be in the range $125<\lambda_{y}^{+} \lesssim 250$. More precisely, figure $13(i, j)$ suggest the threshold should be closer to $\lambda_{y}^{+}=250$ in order to exclude turbulence from the near-wall cycle. We therefore consider the spectral region associated with the Kelvin-Helmholtz instability in our minimal-span channels as $\lambda_{y}^{+} \gtrsim 250$ in the spanwise direction, which is more conservative than the threshold 
$\lambda_{y}^{+} \gtrsim 130$ that García-Mayoral \& Jiménez [14] suggested. In the streamwise direction, the range $65<\lambda_{x}^{+}<290$ [14] is appropriate also for the present data set. This way, we only let fluctuations pass the spectral filter that are due to the Kelvin-Helmholtz instability, while we discard fluctuations associated with the near-wall cycle that characterises both the smooth and riblet wall flows.

\subsection{Dependence of Kelvin-Helmholtz rollers on riblet shape and size}

We now integrate fluctuations in spectral space for only the wavelengths affected by the Kelvin-Helmholtz instability to impose a spectral filter on variance profiles of different flow quantities

$$
{\overline{\phi^{\prime} \psi^{\prime}}}_{K H}^{+}\left(z^{+}\right)=\int_{250}^{\infty}\left[\int_{65}^{290} E_{\phi \psi}^{+} \mathrm{d} \lambda_{x}^{+}\right] \mathrm{d} \lambda_{y}^{+},
$$

where $(\phi \psi) \in\{u u, u w, w \theta\}$. In figure 14, we compare those filtered profiles for all riblet cases to smooth-wall flow. Even though Kelvin-Helmholtz rollers are absent over a smooth wall, $\left({\overline{u^{\prime} u^{\prime}}}_{K H}^{+}\right)^{1 / 2}$ increases over the first 20 viscous units from the wall.

Drag-reducing riblets of any shape have almost the same profile (---- in figure 14, left) as the smooth wall, because they lack Kelvin-Helmholtz rollers. The profiles of Reynolds shear stress (figure 14, centre) and wall-normal transport of the passive scalar (figure 14, right) in the spectral region of Kelvin-Helmholtz rollers are likewise not affected by the presence of small riblets.

For larger, drag-increasing riblets (— in figure 14), differences emerge between riblet shapes. The two drag-increasing triangular riblets with $\alpha=30^{\circ}$ in figure $14(a-c)$ have profiles that are distinctly different from those of the smooth-wall flow. All three quantities peak at $z^{+}-z_{t}^{+}<10$, which is not the case for the smooth wall and therefore indicative of Kelvin-Helmholtz rollers. The distances from the riblet crest match those found by García-Mayoral \& Jiménez [14] for Kelvin-Helmholtz rollers over blade riblets. Indeed, our blades in figure 14(m-o) have similar peaks, that are however lower in magnitude than for the sharp triangular riblets. For the other riblet shapes, fluctuations in the spectral region of Kelvin-Helmholtz rollers resemble more closely those of smooth-wall flow, indicating that the instability is weak or does not develop. For example, we can observe minor effects of the instability for trapezoidal riblets and symmetric triangular riblets with $\alpha=60^{\circ}$, that are much weaker than for the sharper triangular riblets with $\alpha=30^{\circ}$. Here, $\overline{w^{\prime} \theta^{\prime}}{ }_{K H}^{+}$is the most sensitive indicator of all possible cospectra (as also evident in figure 12). The two largest trapezoidal riblets (lightest curves in figure $14 j-l$ ) have higher values of $-{\overline{u^{\prime} w^{\prime}}}_{K H}^{+}$and $-{\overline{w^{\prime} \theta^{\prime}}}_{K H}^{+}$and monotonously increasing profiles not seen for any other riblet, suggesting that structures related to the near-wall cycle influence the spectral region for these very large riblets $\left(s^{+}=50,63\right)$. The instability appears to be absent or vanishingly weak for the triangular riblets with $\alpha=90^{\circ}$ in figure $14(g-i)$ and the asymmetric triangular riblets in figure $14(p-r)$, for which fluctuations in the considered spectral region are almost identical to those over a smooth wall.

The filtered profiles for channels of different width $L_{y}^{+} \approx\{250,450\}$ in figure $14(a-c)$ and in figure 14 $(g-i)$ match within the statistical uncertainty, because energy at $\lambda_{y}^{+}>L_{y}^{+}$is accounted for in the spanwise-infinite wavelength, as also observed in figure 8 at the height $z^{+}-z_{t}^{+}=5$. Reynolds-number effects are negligible, as we can observe for the smooth-wall flows in figure $14(a-c)$ at $R e_{\tau}=395$ and $R e_{\tau}=1000$ respectively and also for the blades in figure $14(m-o)$ at $R e_{\tau}=395$ and $R e_{\tau} \approx 550$. 

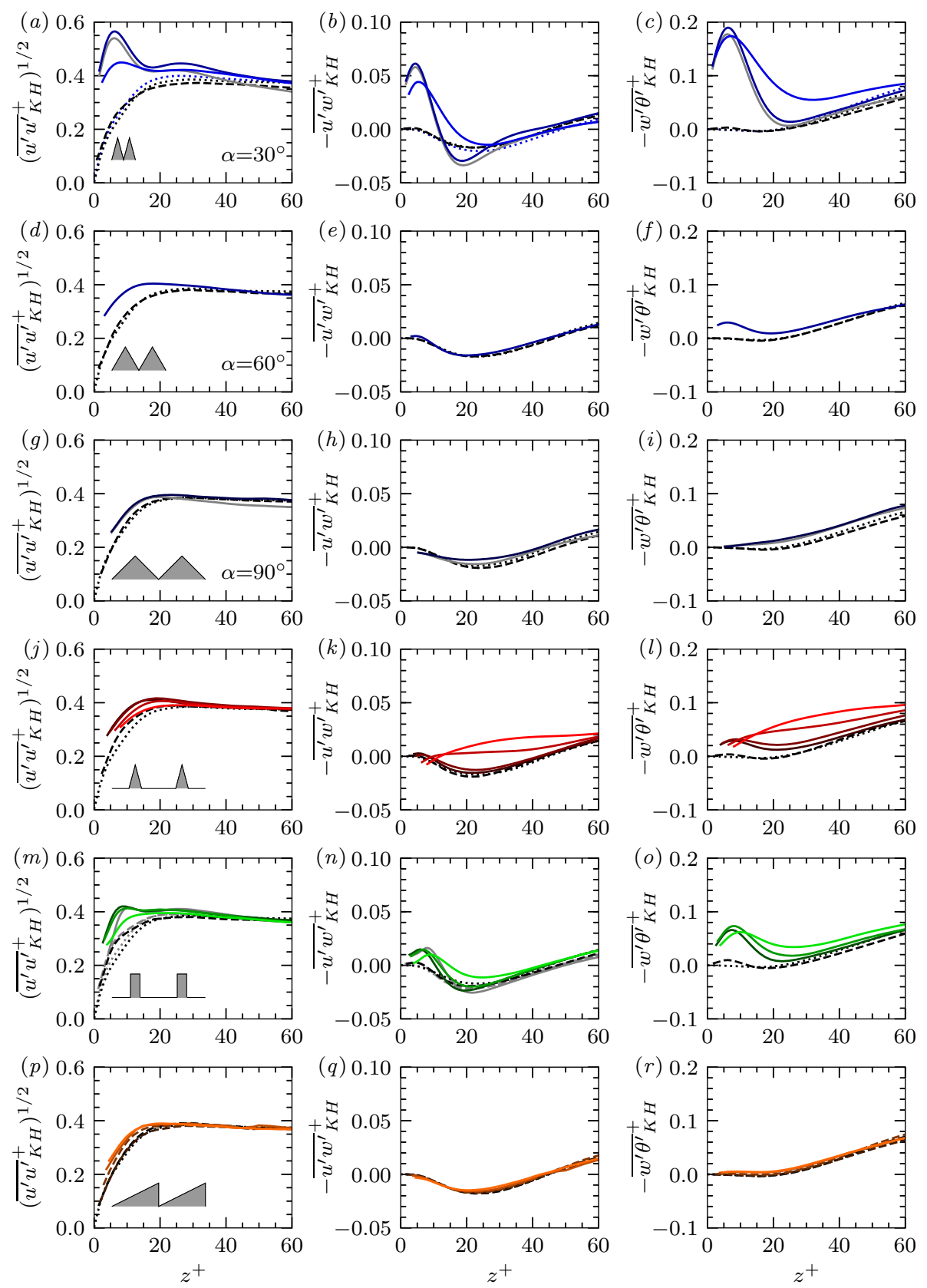

Fig. 14 Kelvin-Helmholtz rollers strongly affect fluctuations in $(a-c),(m-o)$, but not in $(p-r)$ : profiles of streamwise velocity fluctuations (left), Reynolds shear stress (centre) and the wall-normal transport of $\theta$ (right), filtered only for wavelengths associated with the Kelvin-Helmholtz instability as per (10). Smooth wall flow $(\cdots \cdots)$, riblets that reduce $(---)$ or increase $(-)$ drag. Curves for each shape get lighter with increasing riblet size. Light curves in $(a-c)$ are for smooth-wall and riblet flow at $R e_{\tau}=1000$, all others at $R e_{\tau}=395$. Data from the wider domains with $L_{y}^{+} \approx 450$ is shown in grey $(a-c$ and $g-i)$. Grey curves in $(m, n)$ are for reference full-span channel cases $13 \mathrm{~L}$ and $20 \mathrm{~L}$ at $R e_{\tau} \approx 550$ from [15] along with the corresponding smooth-wall profile. 


\section{Conclusion}

Kelvin-Helmholtz rollers have been shown to develop in the flow less than 20 viscous units above blade riblets, where they contribute to drag [14]. In order to investigate effects of the Kelvin-Helmholtz instability on riblets of six different shapes, we applied the minimal-span channel framework for cost-efficient DNS of rough-wall flows [30] to this particular roughness type of streamwise uniform riblets. The minimal domain reduced the computational cost and allowed us to investigate 21 different riblet cases. Since Kelvin-Helmholtz rollers have a larger spanwise extent than turbulence close to a smooth wall [15], we systematically evaluated effects of the minimal domain width $L_{y}^{+} \approx 250$ on large flow structures. Specifically, we compared velocity spectra at different heights to those from minimal channels with $L_{y}^{+}=\{150,450\}$ and also to full-span channel reference data from García-Mayoral \& Jiménez [15]. In agreement with MacDonald et al. [30], we find that large flow structures are unphysically affected down to a lower height than smaller-scale turbulence. Nevertheless, our domains with $L_{y}^{+} \approx 250$ correctly represent fluctuations across all wavelengths, including those of Kelvin-Helmholtz rollers, for at least the first 30 viscous units above the riblet crest.

We re-evaluated the spectral filter by García-Mayoral \& Jiménez [14] for the present riblet geometries to extract variance profiles solely due to the Kelvin-Helmholtz instability. In order to filter fluctuations with sufficient spanwise spectral separation between KelvinHelmholtz rollers and other near-wall turbulence, our domain constraint $L_{y}^{+} \gtrsim 250$ is more stringent than the one determined by MacDonald et al. [30] for general roughness. Comparison to smooth-wall flow, for which the Kelvin-Helmholtz instability is absent, suggests that Kelvin-Helmholtz rollers form in the flow above blade riblets, as shown by García-Mayoral \& Jiménez [14], and also above triangular riblets with a tip angle $\alpha=30^{\circ}$. However, the filtered variance profiles revealed a dependence of Kelvin-Helmholtz rollers on the riblet shape, because the profiles of blunt triangular riblets with $\alpha=60^{\circ}$ to $\alpha=90^{\circ}$ and trapezoidal riblets with $\alpha=30^{\circ}$ resemble that above a smooth wall (figure 14), indicating that these riblet shapes do not trigger the Kelvin-Helmholtz instability.

Acknowledgements The authors gratefully acknowledge the support of the Australian Research Council Discovery Project DP170102595. This research is undertaken with the assistance of resources from NCI that is supported by the Australian Government and from Pawsey that is funded by the Australian Government and the Government of Western Australia. D. Modesti was partially funded by Ermenegildo Zegna, through the "EZ Founder's Scholarship". The authors are grateful to the anonymous reviewers for their helpful comments which improved the quality of the manuscript.

Funding This project was supported by the Australian Research Council Discovery Project DP170102595. D. Modesti was partially funded through the "EZ Founder's Scholarship".

Conflicts of interest The authors declare that they have no conflict of interest.

Code availability Simulations for this project were conducted using the commercial solver Cliff by Cascade Technologies Inc. 


\section{References}

1. Bannier, A., Garnier, É., Sagaut, P.: Riblet flow model based on an extended FIK identity. Flow Turb. Comb. 95(2), 351-376 (2015). DOI 10.1007/s10494-015-9624-2

2. Bechert, D., Bartenwerfer, M.: The viscous flow on surfaces with longitudinal ribs. J. Fluid Mech. 206, 105-129 (1989)

3. Bechert, D., Bruse, M., Hage, W., van der Hoeven, J., Hoppe, G.: Experiments on drag-reducing surfaces and their optimization with an adjustable geometry. J. Fluid Mech. 338, 59-87 (1997)

4. Breugem, W.P., Boersma, B.J., Uittenbogaard, R.E.: The influence of wall permeability on turbulent channel flow. J. Fluid Mech. 562, 35-72 (2006)

5. Chan, L., MacDonald, M., Chung, D., Hutchins, N., Ooi, A.: Secondary motion in turbulent pipe flow with three-dimensional roughness. J. Fluid Mech. 854, 5-33 (2018)

6. Chin, C., Ooi, A.S.H., Marusic, I., Blackburn, H.M.: The influence of pipe length on turbulence statistics computed from direct numerical simulation data. Phys. Fluids 22(11), 115107 (2010)

7. Choi, H., Moin, P., Kim, J.: Direct numerical simulation of turbulent flow over riblets. J. Fluid Mech. 255, 503-539 (1993)

8. Chung, D., Chan, L., MacDonald, M., Hutchins, N., Ooi, A.: A fast direct numerical simulation method for characterising hydraulic roughness. J. Fluid Mech. 773, 418-431 (2015)

9. Drazin, P.G., Reid, W.H.: Hydrodynamic Stability, second edn. Cambridge Mathematical Library (2004)

10. Flores, O., Jiménez, J.: Hierarchy of minimal flow units in the logarithmic layer. Phys. Fluids 22(7), 071704 (2010)

11. Fukagata, K., Iwamoto, K., Kasagi, N.: Contribution of Reynolds stress distribution to the skin friction in wall-bounded flows. Phys. Fluids 14(11), L73-L76 (2002)

12. García-Mayoral, R.: The interaction of riblets with wall-bounded turbulence. Ph.D. thesis, Universidad Politécnica de Madrid (2011)

13. García-Mayoral, R., Gómez-de-Segura, G., Fairhall, C.T.: The control of near-wall turbulence through surface texturing. Fluid Dyn. Res. 51(1), 011410 (2019)

14. García-Mayoral, R., Jiménez, J.: Hydrodynamic stability and breakdown of the viscous regime over riblets. J. Fluid Mech. 678, 317-347 (2011)

15. García-Mayoral, R., Jiménez, J.: Scaling of turbulent structures in riblet channels up to $\operatorname{Re} \tau \approx 550$. Phys. Fluids 24(10), 105101 (2012)

16. Gatti, D., von Deyn, L., Forooghi, P., Frohnapfel, B.: Do riblets exhibit fully rough behaviour? Exp. Fluids (61), 81 (2020)

17. Goldstein, D., Tuan, T.C.: Secondary flow induced by riblets. J. Fluid Mech. 363, 115-151 (1998)

18. Gómez-de-Segura, G., Sharma, A., García-Mayoral, R.: Turbulent drag reduction using anisotropic permeable substrates. Flow Turb. Comb. 100(4), 995-1014 (2018)

19. Ham, F., Mattsson, K., Iaccarino, G.: Accurate and stable finite volume operators for unstructured flow solvers. Center for Turbulence Research, Stanford University/NASA Ames, Annual Research Briefs pp. 243-261 (2006)

20. Ham, F., Mattsson, K., Iaccarino, G., Moin, P.: Towards time-stable and accurate LES on unstructured grids. In: Complex Effects in Large Eddy Simulations, pp. 235-249. Springer Berlin Heidelberg (2007)

21. Hutchins, N., Marusic, I.: Evidence of very long meandering features in the logarithmic region of turbulent boundary layers. J. Fluid Mech. 579, 1-28 (2007)

22. Hwang, Y.: Near-wall turbulent fluctuations in the absence of wide outer motions. J. Fluid Mech. 723, 264-288 (2013)

23. Jiménez, J., Álamo, J.C.D., Flores, O.: The large-scale dynamics of near-wall turbulence. J. Fluid Mech. 505, 179-199 (2004)

24. Jiménez, J., Moin, P.: The minimal flow unit in near-wall turbulence. J. Fluid Mech. 225, 213-240 (1991)

25. Jiménez, J., Uhlmann, M., Pinelli, A., Kawahara, G.: Turbulent shear flow over active and passive porous surfaces. J. Fluid Mech. 442, 89-117 (2001)

26. Lozano-Durán, A., Jiménez, J.: Effect of the computational domain on direct simulations of turbulent channels up to $R e_{\tau}=4200$. Phys. Fluids 26(1), 011702 (2014)

27. Luchini, P.: Reducing the turbulent skin friction. Comp. Methods in App. Sc. 3, 466-470 (1996)

28. Luchini, P., Manzo, F., Pozzi, A.: Resistance of a grooved surface to parallel flow and cross-flow. J. Fluid Mech. 228, 87-109 (1991)

29. MacDonald, M., Chan, L., Chung, D., Hutchins, N., Ooi, A.: Turbulent flow over transitionally rough surfaces with varying roughness densities. J. Fluid Mech. 804, 130-161 (2016)

30. MacDonald, M., Chung, D., Hutchins, N., Chan, L., Ooi, A., García-Mayoral, R.: The minimal-span channel for rough-wall turbulent flows. J. Fluid Mech. 816, 5-42 (2017)

31. Moin, P., Kim, J.: Numerical investigation of turbulent channel flow. J. Fluid Mech. 118, 341-377 (1982) 
32. Moser, R., Kim, J., Mansour, N.: Direct numerical simulation of turbulent channel flow up to $\operatorname{Re}_{\tau}=590$. Phys. Fluids 11(4), 943-945 (1999)

33. Nepf, H.M., Ghisalberti, M., White, B., Murphy, E.: Retention time and dispersion associated with submerged aquatic canopies. Water Resour. Res. 43(4) (2007)

34. Poggi, D., Porporato, A., Ridolfi, L., Albertson, J.D., Katul, G.G.: The effect of vegetation density on canopy sub-layer turbulence. Boundary-Layer Met. 111(3), 565-587 (2004)

35. Raupach, M.R., Finnigan, J.J., Brunet, Y.: Coherent eddies and turbulence in vegetation canopies: The mixing-layer analogy. Boundary-Layer Met. 25, 351-382 (1996)

36. Sharma, A., García-Mayoral, R.: Turbulent flows over dense filament canopies. J. Fluid Mech. 888, A2 (2020)

37. Spalart, P.R., McLean, D.: Drag reduction: enticing turbulence, and then an industry. Phil. Trans. R. Soc. A 369, 1556-1569 (2011)

38. Townsend, A.A.: The Structure of Turbulent Shear Flow, 1 edn. Cambridge University Press (1956)

39. Vinuesa, R., Prus, C., Schlatter, P., Nagib H, M.: Convergence of numerical simulations of turbulent wall-bounded flows and mean cross-flow structure of rectangular ducts. Meccanica 51(12), 3025-3042 (2016)

40. Walsh, M.: Turbulent boundary layer drag reduction using riblets. 20th Aerospace Sciences Meeting (AIAA Paper 1982-169) (1982)

41. Walsh, M., Weinstein, L.: Drag and heat transfer on surfaces with small longitudinal fins. 11th Fluid and Plasma Dynamics Conference (AIAA Paper 1987-1161) (1978) 


\section{University Library}

\section{- M M N E R VA A gateway to Melbourne's research publications}

Minerva Access is the Institutional Repository of The University of Melbourne

Author/s:

Endrikat, S;Modesti, D;MacDonald, M;Garcia-Mayoral, R;Hutchins, N;Chung, D

Title:

Direct Numerical Simulations of Turbulent Flow Over Various Riblet Shapes in Minimal-Span Channels

Date:

2020-11-20

Citation:

Endrikat, S., Modesti, D., MacDonald, M., Garcia-Mayoral, R., Hutchins, N. \& Chung, D. (2020). Direct Numerical Simulations of Turbulent Flow Over Various Riblet Shapes in Minimal-Span Channels. Flow, Turbulence and Combustion, 107 (1), https://doi.org/10.1007/ s10494-020-00224-z.

Persistent Link:

http://hdl.handle.net/11343/252852 